\title{
Briófitas do Parque Estadual da Serra do Mar, Núcleo de Santa Virgínia, Estado de São Paulo, Brasil
}

\author{
Dimas Marchi do Carmo ${ }^{1,2}$, Jéssica Soares de Lima ${ }^{1}$, Leandro de Almeida Amélio ${ }^{1}$ e \\ Denilson Fernandes Peralta ${ }^{1}$
}

Recebido: 16.12.2015; aceito: 20.04.2016

\begin{abstract}
Bryophytes in Parque Estadual da Serra do Mar, Núcleo Santa Virgínia, São Paulo State, Brazil). The Núcleo Santa Virgínia is one of the administrative centers of the extensive Parque Estadual da Serra do Mar that was created in order to protect the rich São Paulo State Atlantic Forest biodiversity. This phytogeographical domain is the richest in bryophyte species and endemic species of the country. Altogether, we found 386 bryophyte species (196 liverworts, 188 mosses, and two hornworts); 32 (8\%) are Brazilian endemic species; 24 new records are registered for São Paulo State and four new records for Atlantic Forest (Cheilolejeunea beyrichii (Lindenb.) Reiner, Microlejeunea acutifolia Steph, Lepidopilum pallidonitens (Müll. Hal.) Paris, and Bryum leptocladon Sull). The liverwort family Lejeuneaceae was the richest with 90 species found in the area, while Pilotrichaceae showed greater richness for mosses, with 20 species. Most species reveal a moderate distribution for the country and are neotropical.
\end{abstract}

Keywords: Atlantic Forest, biodiversity, hornworts, liverworts, mosses

RESUMO - (Briófitas do Parque Estadual da Serra do Mar, Núcleo Santa Virgínia, Estado de São Paulo, Brasil). O Núcleo Santa Virgínia é um dos centros administrativos do extenso Parque Estadual da Serra do Mar que foi criado para proteger a rica biodiversidade da Mata Atlântica do Estado de São Paulo. Esse domínio fitogeográfico possui a maior riqueza e endemismo de espécies de briófitas do país. Foram encontradas 386 espécies de briófitas (196 hepáticas, 188 musgos e dois antóceros), $32(8 \%)$ espécies endêmicas brasileiras, 24 novos registros para o Estado de São Paulo e quatro novas ocorrências para a Mata Atlântica (Cheilolejeunea beyrichii (Lindenb.) Reiner, Microlejeunea acutifolia Steph, Lepidopilum pallidonitens (Müll. Hal.) Paris e Bryum leptocladon Sull.). A família de hepáticas Lejeuneaceae foi a mais rica, com 90 espécies, enquanto Pilotrichaceae apresentou maior riqueza para os musgos, com 20 espécies. A maioria das espécies encontradas apresenta uma distribuição moderada para o país e são neotropicais.

Palavras-chave: antóceros, Mata Atlântica, biodiversidade, hepáticas, musgos

\section{Introdução}

O Parque Estadual da Serra do Mar (PESM) é uma unidade de conservação criada em 1977 pelo Decreto $\mathrm{n}^{\circ} 10.251(30 / 08 / 1977)$ com o intuito de preservar a rica biodiversidade de fauna e flora existente nos ameaçados domínios da Mata Atlântica. Com 332.690 ha, ele representa a maior unidade de conservação de Mata Atlântica existente no país, além disso, em virtude de seu extenso território e pela vasta heterogeneidade sociocultural e de paisagens naturais existentes, atualmente o PESM é administrado por vários núcleos independentes que se encontram em diferentes fases de implantação (Instituto Ekos Brasil 2006, SMA 2015).
O Núcleo Santa Virgínia do PESM desenvolve diversos tipos de atividades voltadas à conservação do parque, trabalhando em pesquisas científicas nas áreas de biodiversidade e ecologia, além de atuar em projetos educacionais voltados à conscientização ambiental e importância das florestas da Mata Atlântica (Pires 2015). Ele abrange uma área de 17.500 ha. e foi criado a partir de desapropriações da fazenda Santa Virgínia (que deu origem ao nome), sendo $68 \%$ de suas terras de domínio público e $32 \%$ pertencentes ao domínio privado. O principal propósito da criação desse Núcleo consta na proteção da maior parte das florestas intactas da região do Vale do Paraíba e do Rio Paraibuna, importante corpo

1. Instituto de Botânica, Núcleo de Pesquisa em Briologia, Caixa Postal 68041, 04045-972 São Paulo, SP, Brasil

2. Autor para correspondência: dimas.botanica@gmail.com 
hídrico formador de corredeiras e cachoeiras dentro do PESM (SMA 2015).

Em relação aos estudos de briófitas feitos para a Mata Atlântica ao longo de toda a costa litorânea brasileira, temos uma ampla bibliografia disponível, com vários trabalhos tanto com enfoques florísticos quanto ecológicos (Costa \& Lima 2005, Valente \& Pôrto 2006, Santos \& Costa 2008, Costa \& Santos 2009, Santos \& Costa 2010a, b, Silva \& Pôrto 2010, Costa et al. 2015 e Silva \& Pôrto 2015). A Mata Atlântica é o domínio fitogeográfico que apresenta as maiores taxas de endemismo e de riqueza de briófitas para o país (Costa et al. 2011), mesmo com apenas 11\% de sua vegetação remanescente, ela ainda é um dos domínios mais ameaçados pelas pressões antrópicas e considerada um hotspot mundial (Fundação SOS Mata Atlântica 2015).

Para o Estado de São Paulo encontramos uma série de trabalhos com briófitas ocorrentes em regiões da Mata Atlântica, (Visnadi 2002, Visnadi 2004, Visnadi 2005, Visnadi 2006, Yano \& Peralta 2007, Peralta \& Yano 2008, Yano \& Peralta 2008, Visnadi 2009, Peralta \& Yano 2012 e Visnadi 2013), os quais contribuíram com o conhecimento da distribuição e ocorrência de briófitas, resultando em dados compilados atualmente de 890 espécies ocorrendo na Mata Atlântica, o que representa ca. de $99 \%$ das briófitas totais estimadas para o Estado (Costa \& Peralta 2015).

O conhecimento da biodiversidade de briófitas auxilia, e justifica, a manutenção das unidades de conservação, bem como estabelece meios de divulgação de informação sobre a diversidade de briófitas brasileiras (Visnadi 2005, Peralta \& Yano 2012). Sendo assim, o objetivo desse estudo foi apresentar uma lista das espécies ocorrentes no Núcleo Santa Virgínia dentro da Mata Atlântica e, dessa forma, contribuir ainda mais com os estudos de briófitas sobre sua distribuição geográfica brasileira, os domínios fitogeográficos que ocupam e sua relação com a distribuição mundial, visto que, muitas vezes, essas informações carecem em outros trabalhos desenvolvidos para essa área e, até o momento, ainda não existem inventários florísticos de briófitas dentro dessa importante parcela da reserva.

\section{Material e métodos}

O Núcleo Santa Virgínia do PESM abrange os municípios de São Luiz do Paraitinga e Natividade da Serra, localizando-se aproximadamente nas coordenadas geográficas $45^{\circ} 03^{\prime}-45^{\circ} 11^{\prime} \mathrm{O}$ e $23^{\circ} 17^{\prime}$ $23^{\circ} 24^{\prime}$ S. Possui uma área total de 17.000 ha, amplitude altitudinal de $860 \mathrm{~m}$ a $1.650 \mathrm{~m}$ e temperaturas média de $21{ }^{\circ} \mathrm{C}$, com máxima de $35{ }^{\circ} \mathrm{C}$, e mínima de $-3{ }^{\circ} \mathrm{C}$ (Pires 2015). A sua vegetação é composta basicamente pela Floresta Ombrófila Densa Montana e Floresta Ombrófila Densa Alto Montana, com alguns pequenos trechos de Campos de Altitude e de Floresta de Neblina (SMA 2015).

Foram realizadas expedições de coletas nos anos de 2013 e análises de materiais depositados no herbário do Instituto de Botânica Maria Eneyda Pacheco Kauffman Fidalgo (SP). Foram estudadas 1918 amostras (exsicatas) provenientes do PESM no Núcleo Santa Virgínia. As coletas foram realizadas por meio de caminhadas livres e em todos os tipos de substratos disponíveis que as briófitas pudessem colonizar. A metodologia para a coleta, herborização e preservação do material seguiu Gradstein et al. (2001) e todo o material obtido nas coletas está depositado no herbário SP.

As referências usadas para a identificação das espécies foram Frahm (1991), Sharp et al. (1994), Buck (1998), Gradstein et al. (2001), Gradstein \& Costa (2003), Câmara \& Costa (2006), Câmara (2008a, b), Ballejos \& Bastos (2009), Yano \& Peralta (2009), Yano \& Peralta (2011) e Bordin \& Yano (2013). Os sistemas de classificação adotados foram Renzaglia et al. (2009) para Anthocerotophyta, Crandall-Stotler et al. (2009) para Marchantiophyta e Goffinet et al. (2009) para Bryophyta.

A lista das espécies está organizada em ordem alfabética por divisão, família, gênero e espécie. A distribuição geográfica brasileira das espécies é apresentada discriminada em ampla, moderada e rara seguindo a metodologia utilizada por Valente \& Pôrto (2006), bem como a distribuição mundial e dos domínios fitogeográficos brasileiros que as briófitas ocupam, conforme Gradstein \& Costa (2003), Yano (2008), Forzza et al. (2010), Costa et al. (2011) e Costa \& Peralta (2015).

\section{Resultados e Discussão}

Foram encontradas 386 espécies distribuídas em 57 famílias e 24 novas ocorrências de briófitas para o Estado de São Paulo. A divisão dos musgos apresentou 37 famílias e 188 espécies; as hepáticas resultaram em 19 famílias e 196 espécies, enquanto para os antóceros 
foram encontradas apenas duas espécies representadas em apenas uma família (tabela 1). Esse número de espécies representa $43 \%$ das briófitas registradas para o estado de São Paulo, 25\% das registradas para o Brasil e $10 \%$ das encontradas para a América tropical (Gradstein et al. 2001, Costa \& Peralta 2015) (tabela 2).

Para os musgos, a família Pilotrichaceae teve maior riqueza apresentada, sendo encontradas 20 espécies, enquanto as famílias Fissidentaceae e Sematophyllaceae apresentaram 15 espécies, Bryaceae e Orthotrichaceae 12 espécies e Leucobryaceae com 11 espécies (figura 1). A região sudeste do país pode ser considerada um dos centros de diversidade para a família Pilotrichaceae (Vaz \& Costa 2006), conforme os resultados desse estudo, essa informação é corroborada pela elevada riqueza e número de novas ocorrências encontradas. Em outros trabalhos realizados no estado de São Paulo, essa família também foi bem representativa, esteve entre as mais abundantes e ricas (Visnadi 2005, Visnadi 2009, Yano \& Peralta 2007, Peralta \& Yano 2008) bem como as famílias Fissidentaceae, Sematophyllaceae, Bryaceae, Orthotrichaceae e Leucobryaceae. Em levantamentos florísticos realizados em outras áreas de Mata Atlântica pelo país, essas famílias de musgos também estão entre as mais representativas (Santos \& Costa 2008, Valente et al. 2009, Costa et al. 2015 e Silva \& Pôrto 2015).

A família Lejeuneaceae foi a família de hepáticas que apresentou a maior riqueza, com 90 espécies (figura 2), o que representa ca. de $46 \%$ das hepáticas encontradas nesse trabalho, destacou-se também pelo número de amostras que obteve, com 664 espécimes e representando ca. de $35 \%$ do total. Para o Brasil são relacionadas aproximadamente 285 espécies de Lejeuneaceae distribuídas em 55 gêneros (Costa et al. 2015), além disso, essa família também foi a mais rica em demais trabalhos nos estados de São Paulo (Visnadi 2005, Visnadi 2009; Yano \& Peralta 2007, Peralta \& Yano 2008), bem como para outras áreas pelo Brasil (Valente \& Pôrto 2006, Santos \& Costa 2008, Costa et al. 2015, Reis et al. 2015 e Silva \& Pôrto 2015). Ela é muito bem representada na Mata Atlântica em virtude do clima úmido e da presença de muitos substratos disponíveis onde essas espécies podem crescer, desde galhos, troncos de árvores vivas ou caídas, até rochas, solos e folhas vivas (Gradstein et al. 2001).

Para os antóceros, apenas a família Dendrocerotaceae foi encontrada, apresentando as espécies Dendroceros crispus (Sw.) Nees e Nothoceros minarum (Ness) J.C.
Villarreal., as quais foram encontradas crescendo em diferentes substratos no Núcleo Santa Virgínia nos dois municípios.

Em relação à distribuição geográfica das espécies encontradas no Brasil do Núcleo Santa Virgínia, observamos que 166 espécies (43\%) apresentaram uma distribuição considerada moderada (Valente \& Pôrto 2006), ou seja, ocorriam de cinco a nove Estados brasileiros, enquanto para uma distribuição ampla (as que ocorrem em 10 ou mais Estados brasileiros) foram encontradas 131 espécies (34\%) e 89 espécies (23\%) apresentaram uma distribuição rara ou mais restrita (1-4 Estados brasileiros) (tabela 1). Quando aumentamos a escala da distribuição geográfica para um nível mais global, observamos a predominância de espécies neotropicais, com 146 espécies (38\%), 123 espécies (32\%) ocorrendo na América tropical, 35 espécies $(9 \%)$ pantropicais e apenas $27(7 \%)$ cosmopolitas (tabela 1).

Entre as briófitas endêmicas do Brasil, 32 espécies (8\%) foram encontradas no Núcleo Santa Virgínia, sendo entre essas espécies quatro registradas como novas ocorrências para o Estado de São Paulo, identificadas como Bryum subapiculatum Hampe, Schlotheimia elata Mitt., Lepidopilum caudicaule Müll. Hal. e Orthostichopsis latifolia Sehnem.

Foram encontradas ao todo 24 espécies (6\%) como novas ocorrências para o Estado de São Paulo, das quais 21 apresentaram uma distribuição rara pelo país, enquanto as três restantes uma distribuição moderada; 11 espécies ocorrem no Neotrópico, seis se distribuem para a América tropical, uma pantropical (Brachythecium ruderale (Brid.) Buck) e duas são cosmopolitas (Bryum muehlenbeckii Bruch \& Schimp. e Hedwigidium integrifolium (P. Beauv.) Dixon) (tabela 1). Esses resultados ressaltam a importância dos trabalhos de levantamentos florísticos para o conhecimento da biodiversidade de briófitas existentes nos domínios da Mata Atlântica para o Estado de São Paulo, bem como estão de acordo com conclusões de outros trabalhos já feitos para esse mesmo domínio (Yano \& Peralta 2008, Peralta \& Yano 2012).

A Mata Atlântica é o domínio mais rico de espécies de briófitas existente no país (Costa et al. 2011), sendo assim, o Núcleo Santa Virgínia, e outras reservas existentes para sua proteção, garantem a importância de manter essas áreas como locais de conservação e reservatórios de diversidade no Estado de São Paulo (Visnadi 2005). De acordo com os resultados desse estudo, 382 (99\%) das espécies encontradas ocorrem na Mata Atlântica, sendo que 
Tabela 1. Lista das espécies ocorrentes no Parque Estadual da Serra do Mar, Núcleo de Santa Virgínia, Estado de São Paulo, Brasil. AM: Amazônia, CA: Caatinga, CE: Cerrado, MA: Mata Atlântica, PM: Pampa, PN: Pantanal. Distr. Brasil (Distribuição brasileira). Distr. Mundial (Distribuição mundial). *: Nova ocorrência para o estado de São Paulo.

Table 1. List of species occurring in the State Park of Serra do Mar, Núcleo Santa Virgínia, São Paulo State, Brazil. AM: Amazon, CA: Caatinga, CE: Cerrado, MA: Atlantic Forest, PM: Pampa, PN: Pantanal. Distr. Brasil (Brazilian Distribution). Distr. Mundial (Worldwide distribution). *: New occurrence for the State of São Paulo.

\begin{tabular}{|c|c|c|c|c|}
\hline Táxons (Divisão/Família/Espé & $\begin{array}{c}\text { Domínio } \\
\text { Fitogeográfico }\end{array}$ & $\begin{array}{c}\text { Distribuição } \\
\text { Brasil }\end{array}$ & $\begin{array}{c}\text { Distribuição } \\
\text { mundial }\end{array}$ & Voucher \\
\hline
\end{tabular}

Anthocerotophyta

Dendrocerotaceae

Dendroceros crispus (Sw.) Nees

Nothoceros minarum (Ness)

J.C. Villarreal

Bryophyta

Adelotheciaceae

Adelothecium bogotense (Hampe) Mitt.

MA, PM

Moderada

Bartramiaceae

*Breutelia tomentosa (Sw. ex Brid.)

A. Jaeger

Leiomela aristifolia (A. Jaeger)

Wijk \& Margad.

Leiomela bartramioides (Hook.) Paris

Philonotis elongata (Dism.) H.A.

Crum \& Steere

MA

Rara

MA

MA

$\mathrm{AM}, \mathrm{CE}, \mathrm{MA}$

Moderada

Philonotis sphaerocarpa (Hedw.) Brid.

Philonotis uncinata (Schwägr.) Brid.

Brachytheciaceae

Aerolindigia capillacea (Hornsch.) M. Menzel

*Brachythecium ruderale (Brid.) Buck

Helicodontium capillare (Hedw.)

A. Jaeger

Meteoridium remotifolium (Müll. Hal.) Manuel

Platyhypnidium aquaticum (A. Jaeger)

M. Fleisch.

Rhynchostegium scariosum (Taylor) A. Jaeger

*Rhynchostegium serrulatum (Hedw.) A. Jaeger

Squamidium brasiliense Broth.

Zelometeorium ambiguum (Hornsch.) Manuel

$\mathrm{AM}, \mathrm{CA}, \mathrm{CE}, \mathrm{MA}$

$\mathrm{AM}, \mathrm{CA}, \mathrm{CE}, \mathrm{MA}$, $\mathrm{PM}, \mathrm{PN}$

Moderada

Ampla

MA

Rara

MA, PN

Moderada

AM, CE, MA, PM

Ampla

AM, CE, MA

Ampla

AM, MA

Moderada

CE, MA, PN

Moderada

MA

MA

Rara

Moderada

AM, MA

Moderada

Zelometeorium patulum (Hedw.) Manuel
$\mathrm{AM}, \mathrm{CE}, \mathrm{MA}, \mathrm{PN}$
Ampla
Neotropical

Peralta \& Gugliota 13565

Peralta \& Gugliota 13520

Carmo \& Peralta 550

Peralta et al. 9327

Peralta \& Carmo 14672

Carmo \& Peralta 710

Carmo \& Peralta 545

Américas

Cosmopolita

Peralta et al. 9379

Peralta \& Gugliota 13775

Peralta \& Gugliota 13801

Peralta \& Carmo 14607

Peralta et al. 9415

Carmo \& Peralta 763

Peralta et al. 9343

Peralta et al. 9305

Peralta \& Carmo 14196

América tropical e África

América do Sul e

África

Neotropical Carmo \& Peralta 708
Peralta et al. 9034

Peralta et al. 8936 
Tabela 1 (continuação)

\begin{tabular}{|c|c|c|c|c|}
\hline Táxons (Divisão/Família/Espécie) & $\begin{array}{c}\text { Domínio } \\
\text { Fitogeográfico }\end{array}$ & $\begin{array}{l}\text { Distribuição } \\
\text { Brasil }\end{array}$ & $\begin{array}{c}\text { Distribuição } \\
\text { mundial }\end{array}$ & Voucher \\
\hline \multicolumn{5}{|l|}{ Bryaceae } \\
\hline $\begin{array}{l}\text { Brachymenium consimile (Mitt.) A. } \\
\text { Jaeger }\end{array}$ & MA & Rara & Neotropical & Carmo \& Peralta 567 \\
\hline Brachymenium hornschuchianum Mart. & MA & Moderada & $\begin{array}{l}\text { Endêmica do } \\
\text { Brasil }\end{array}$ & Peralta et al. 9077 \\
\hline $\begin{array}{l}\text { Brachymenium radiculosum (Schwägr.) } \\
\text { Hampe }\end{array}$ & $\mathrm{CE}, \mathrm{MA}$ & Moderada & Neotropical & Peralta et al. 8912 \\
\hline Bryum apiculatum Schwägr. & $\mathrm{AM}, \mathrm{CA}, \mathrm{CE}, \mathrm{MA}$ & Ampla & Cosmopolita & $\begin{array}{c}\text { Peralta \& Gugliota } \\
13874\end{array}$ \\
\hline Bryum argenteum Broth. & $\begin{array}{c}\mathrm{AM}, \mathrm{CA}, \mathrm{CE}, \mathrm{MA} \\
\mathrm{PM}\end{array}$ & Ampla & Cosmopolita & $\begin{array}{l}\text { Peralta \& Carmo } \\
\text { s.n.(SP 438824) }\end{array}$ \\
\hline Bryum densifolium Brid. & $\mathrm{MA}, \mathrm{CE}$ & Ampla & Neotropical & Carmo \& Peralta 560 \\
\hline Bryum huillense Welm. \& Duby & MA & Rara & Cosmopolita & Peralta et al. 9350 \\
\hline *Bryum leptocladon Sull. & $\mathrm{CE}$ & Rara & Neotropical & $\begin{array}{c}\text { Peralta \& Gugliota } \\
13778\end{array}$ \\
\hline Bryum limbatum Müll. Hal. & $\mathrm{MA}, \mathrm{CE}$ & Moderada & Neotropical & Carmo \& Peralta 731 \\
\hline *Bryum muehlenbeckii Bruch \& Schimp. & MA & Rara & Cosmopolita & Peralta et al. 9284 \\
\hline $\begin{array}{l}\text { Bryum renauldii Röll ex Renauld \& } \\
\text { Cardot }\end{array}$ & MA & Rara & Neotropical & $\begin{array}{l}\text { Peralta \& Carmo } \\
\text { s.n.(SP 438826) }\end{array}$ \\
\hline *Byum subapiculatum Hampe & MA & Rara & $\begin{array}{l}\text { Endêmica do } \\
\text { Brasil }\end{array}$ & $\begin{array}{c}\text { Peralta \& Carmo } \\
14352\end{array}$ \\
\hline \multicolumn{5}{|l|}{ Calymperaceae } \\
\hline Syrrhopodon cymbifolius Müll. Hal. & $\mathrm{AM}, \mathrm{CE}, \mathrm{MA}$ & Moderada & $\begin{array}{l}\text { América do Sul } \\
\text { tropical }\end{array}$ & $\begin{array}{c}\text { Peralta \& Carmo } \\
14472\end{array}$ \\
\hline Syrrhopodon gaudichaudii Mont. & $\begin{array}{c}\mathrm{AM}, \mathrm{CA}, \mathrm{CE}, \mathrm{MA} \\
\mathrm{PN}\end{array}$ & Ampla & Pantropical & $\begin{array}{c}\text { Peralta \& Gugliota } \\
13864\end{array}$ \\
\hline Syrrhopodon parasiticus (Brid.) Besch. & $\mathrm{AM}, \mathrm{CE}, \mathrm{MA}, \mathrm{PN}$ & Ampla & Pantropical & Peralta et al. 8878 \\
\hline Syrrhopodon prolifer Schwägr. & $\mathrm{AM}, \mathrm{CA}, \mathrm{CE}, \mathrm{MA}$ & Ampla & Pantropical & Carmo \& Peralta 707 \\
\hline Syrrhopodon tortilis Hampe & MA & Moderada & Neotropical & $\begin{array}{c}\text { Peralta \& Carmo } \\
14744\end{array}$ \\
\hline \multicolumn{5}{|l|}{ Cryphaeaceae } \\
\hline $\begin{array}{l}\text { Schoenobryum concavifolium (Griff.) } \\
\text { Gangulee }\end{array}$ & $\begin{array}{c}\mathrm{AM}, \mathrm{CE}, \mathrm{MA}, \mathrm{PM} \\
\text { PN }\end{array}$ & Ampla & Cosmopolita & Carmo \& Peralta 568 \\
\hline \multicolumn{5}{|l|}{ Daltoniaceae } \\
\hline $\begin{array}{l}\text { Calyptrochaeta albescens (Hampe) } \\
\text { Buck }\end{array}$ & MA & Rara & $\begin{array}{l}\text { Endêmica do } \\
\text { Brasil }\end{array}$ & $\begin{array}{c}\text { Peralta \& Gugliota } \\
13495\end{array}$ \\
\hline *Daltonia bilimbata Hampe & MA & Rara & Neotropical & $\begin{array}{l}\text { Peralta \& Carmo s.n. } \\
\quad \text { (SP 438832) }\end{array}$ \\
\hline Daltonia brasiliensisMitt. & MA & Rara & $\begin{array}{l}\text { Andes tropical e } \\
\text { Brasil }\end{array}$ & Peralta et al. 8925 \\
\hline Daltonia gracilisMitt. & MA & Moderada & Neotropical & $\begin{array}{c}\text { Peralta \& Gugliota } \\
13469\end{array}$ \\
\hline $\begin{array}{l}\text { Daltonia splachnoides (Sm.) Hook. \& } \\
\text { Taylor }\end{array}$ & MA & Moderada & Neotropical & $\begin{array}{c}\text { Peralta \& Carmo } \\
14314\end{array}$ \\
\hline Daltonia stenophylla Mitt. & MA & Rara & Neotropical & Peralta et al. 8934 \\
\hline
\end{tabular}


Tabela 1 (continuação)

\begin{tabular}{ccccc}
\hline Táxons (Divisão/Família/Espécie) & $\begin{array}{c}\text { Domínio } \\
\text { Fitogeográfico }\end{array}$ & $\begin{array}{c}\text { Distribuição } \\
\text { Brasil }\end{array}$ & $\begin{array}{c}\text { Distribuição } \\
\text { mundial }\end{array}$ & Voucher \\
\hline $\begin{array}{c}\text { Leskeodon aristatus (Geh. \& Hampe) } \\
\text { Broth. }\end{array}$ & MA & Moderada & $\begin{array}{c}\text { Endêmica do } \\
\text { Brasil }\end{array}$ & $\begin{array}{c}\text { Peralta \& Carmo } \\
14201\end{array}$
\end{tabular}

Dicranaceae

Holomitrium crispulum Mart.

Holomitrium longifolium Hampe

Leucoloma cruegerianum (Müll. Hal.)

A. Jaeger

$\begin{array}{cccc}\text { AM, CE, MA, PN } & \text { Ampla } & \text { Neotropical } & \text { Peralta et al. } 9380 \\ \text { MA } & \text { Rara } & \text { Neotropical } & \text { Peralta } \text { et al. } 8870 \\ \text { CE, MA } & \text { Moderada } & \text { Neotropical } & \text { Carmo \& Peralta } 748 \\ \text { MA } & \text { Moderada } & \text { Neotropical } & \text { Carmo \& Peralta } 607 \\ \text { MA } & \text { Rara } & \text { Endêmica do } & \text { Carmo \& Peralta } 782\end{array}$

Leucoloma triforme (Mitt.) A. Jaeger

Diphysiaceae

Diphyscium longifolium Griff.

AM, CE, MA Moderada Pantropical Peralta et al. 8981

Fissidentaceae

*Fissidens amoenus Müll. Hal.

Fissidens asplenioides Hedw.

Fissidens bryoides Hedw.

MA

MA, CE

MA

Fissidens elegans Brid.

Fissidens goyazensis Broth.

Fissidens hornschuchii Mont.

Fissidens lagenarius Mitt.

Fissidens oediloma Müll. Hal. ex Broth.

Fissidens pellucidus Hornsch.

Fissidens pseudoplurisetusJ. Bordin, Pursell \& O. Yano

Fissidens saprophilus Broth.

Fissidens scariosus Mitt.

Fissidens serratus Müll. Hal.

Fissidens weirii Mitt.

Fissidens yanoae Pursell

Hedwigiaceae

*Hedwigidium integrifolium (P. Beauv.) Dixon

Hookeriaceae

Hookeria acutifolia Hook. \& Grev.

Hylocomiaceae

Puiggariopsis aurifolia (Mitt.) M. Menzel

\begin{tabular}{|c|c|c|c|}
\hline & Rara & $\begin{array}{c}\text { América do Sul e } \\
\text { EUA }\end{array}$ & Peralta et al. 9412 \\
\hline $\begin{array}{c}\mathrm{AM}, \mathrm{CA}, \mathrm{CE}, \mathrm{MA}, \\
\text { PM, PN }\end{array}$ & Ampla & Neotropical & Peralta et al. 9019 \\
\hline $\mathrm{AM}, \mathrm{CA}, \mathrm{CE}, \mathrm{MA}$ & Ampla & Neotropical & $\begin{array}{c}\text { Peralta \& Gugliota } \\
13529\end{array}$ \\
\hline $\begin{array}{l}\text { AM, CA, CE, MA, } \\
\text { PM, PN }\end{array}$ & Ampla & Neotropical & Peralta et al. 9023 \\
\hline $\begin{array}{c}\mathrm{AM}, \mathrm{CA}, \mathrm{CE}, \mathrm{MA}, \\
\text { PN }\end{array}$ & Ampla & Neotropical & $\begin{array}{c}\text { Peralta \& Gugliota } \\
13615\end{array}$ \\
\hline MA & Moderada & Brasil e Paraguai & $\begin{array}{c}\text { Peralta \& Carmo } \\
14787\end{array}$ \\
\hline $\begin{array}{c}\mathrm{AM}, \mathrm{CA}, \mathrm{CE}, \mathrm{MA}, \\
\text { PM, PN }\end{array}$ & Ampla & Neotropical & Carmo \& Peralta 714 \\
\hline MA & Rara & $\begin{array}{l}\text { Endêmica do } \\
\text { Brasil }\end{array}$ & Peralta et al. 9014 \\
\hline MA & Rara & Brasil e Paraguai & Carmo \& Peralta 615 \\
\hline $\mathrm{AM}, \mathrm{CE}, \mathrm{MA}$ & Ampla & Neotropical & $\begin{array}{c}\text { Peralta \& Gugliota } \\
13541\end{array}$ \\
\hline $\mathrm{AM}, \mathrm{CA}, \mathrm{CE}, \mathrm{MA}$ & Ampla & Neotropical & Carmo \& Peralta 655 \\
\hline CE, MA & Moderada & Neotropical & $\begin{array}{c}\text { Peralta \& Gugliota } \\
13667\end{array}$ \\
\hline MA & Rara & $\begin{array}{l}\text { Endêmica do } \\
\text { Brasil }\end{array}$ & $\begin{array}{c}\text { Peralta \& Gugliota } \\
13464\end{array}$ \\
\hline MA & Moderada & Cosmopolita & Peralta et al. 9393 \\
\hline MA & Moderada & Cosmopolita & Peralta et al. 9397 \\
\hline MA & Rara & Neotropical & $\begin{array}{c}\text { Peralta \& Gugliota } \\
13769\end{array}$ \\
\hline
\end{tabular}

Carmo \& Peralta 522

Peralta \& Gugliota 13767

Pantropical

ca do Sul e

EUA

Peralta et al. 9412

Peralta et al. 9019

\& Gugliota

Peralta et al. 9023

Peralta \& Gugliota

Peralta \& Carmo 14787

$\mathrm{AM}, \mathrm{CA}, \mathrm{CE}, \mathrm{MA}$

dêmica do

Carmo \& Peralta 615

\& Gugliota

Carmo \& Peralta 655

13667

13464

13769

continua 
Tabela 1 (continuação)

\begin{tabular}{|c|c|c|c|c|}
\hline Táxons (Divisão/Família/Espécie) & $\begin{array}{c}\text { Domínio } \\
\text { Fitogeográfico } \\
\end{array}$ & $\begin{array}{c}\text { Distribuição } \\
\text { Brasil }\end{array}$ & $\begin{array}{c}\text { Distribuição } \\
\text { mundial }\end{array}$ & Voucher \\
\hline \multicolumn{5}{|l|}{ Hypnaceae } \\
\hline $\begin{array}{l}\text { Crhysohypnum diminutivum (Hampe) } \\
\text { Buck }\end{array}$ & $\begin{array}{l}\text { AM, CE, MA, PM, } \\
\text { PN }\end{array}$ & Ampla & Cosmopolita & Carmo \& Peralta 726 \\
\hline $\begin{array}{l}\text { Chrysohypnum elegantulum (Hook.) } \\
\text { Hampe }\end{array}$ & $\mathrm{AM}, \mathrm{CE}, \mathrm{MA}, \mathrm{PN}$ & Ampla & Neotropical & $\begin{array}{c}\text { Peralta \& Gugliota } \\
13437\end{array}$ \\
\hline $\begin{array}{l}\text { Mittenothamnium reduncum (Schimp. } \\
\text { ex Mitt.) Ochyra }\end{array}$ & MA & Moderada & Neotropical & $\begin{array}{c}\text { Peralta \& Carmo } \\
14502\end{array}$ \\
\hline $\begin{array}{l}\text { Mittenothamnium reptans (Hampe) } \\
\text { Cardot }\end{array}$ & CE, MA, PM & Ampla & Neotropical & $\begin{array}{c}\text { Peralta \& Gugliota } \\
13550\end{array}$ \\
\hline $\begin{array}{l}\text { Taxiphyllum taxirameum (Mitt.) M. } \\
\text { Fleisch. }\end{array}$ & CE, MA, PN & Moderada & Neotropical & Carmo \& Peralta 793 \\
\hline $\begin{array}{l}\text { Vesicularia vesicularis (Schwägr.) } \\
\text { Broth. }\end{array}$ & AM, CE, MA, PN & Ampla & Neotropical & Carmo \& Peralta 538 \\
\hline \multicolumn{5}{|l|}{ Hypopterygiaceae } \\
\hline $\begin{array}{l}\text { Hypopterygium tamariscina (Hedw.) } \\
\text { Brid. ex Müll. Hal. }\end{array}$ & MA & Moderada & Cosmopolita & Peralta et al. 9293 \\
\hline Lopidium concinnum (Hook.) Wilson & AM, MA & Moderada & Hemisfério sul & Peralta et al. 9012 \\
\hline \multicolumn{5}{|l|}{ Lembophyllaceae } \\
\hline $\begin{array}{l}\text { Orthostichella pachygastrella (Müll. } \\
\text { Hal.) Allen \& Magill }\end{array}$ & MA & Moderada & Neotropical & Peralta et al. 8963 \\
\hline $\begin{array}{l}\text { Orthostichella versicolor (Müll. Hal.) } \\
\text { Allen \& Buck }\end{array}$ & AM, MA & Ampla & $\begin{array}{l}\text { Neotropical e } \\
\text { África }\end{array}$ & Peralta et al. 9337 \\
\hline $\begin{array}{l}\text { Orthostichela welwitschii (Duby) B.H. } \\
\text { Allen \& Magill }\end{array}$ & MA & Rara & $\begin{array}{l}\text { América do Sul e } \\
\text { África }\end{array}$ & $\begin{array}{c}\text { Peralta \& Carmo } \\
14654\end{array}$ \\
\hline Pilotrichella flexilis (Hedw.) Ångstr. & AM, CE, MA, PN & Ampla & $\begin{array}{l}\text { Neotropical e } \\
\text { África }\end{array}$ & $\begin{array}{c}\text { Peralta \& Carmo } \\
14734\end{array}$ \\
\hline \multicolumn{5}{|l|}{ Leucobryaceae } \\
\hline $\begin{array}{l}\text { Campylopus arctocarpus (Hornsch.) } \\
\text { Mitt. }\end{array}$ & CA, CE, MA, PM & Ampla & Pantropical & Peralta et al. 8828 \\
\hline $\begin{array}{l}\text { Campylopus dichrostis (Müll. Hal.) } \\
\text { Paris }\end{array}$ & $\mathrm{MA}, \mathrm{CE}$ & Moderada & $\begin{array}{l}\text { Endêmica do } \\
\text { Brasil }\end{array}$ & Peralta et al. 8909 \\
\hline Campylopus filifolius (Hornsch.) Mitt. & $\mathrm{AM}, \mathrm{CA}, \mathrm{MA}$ & Ampla & Neotropical & Carmo \& Peralta 555 \\
\hline $\begin{array}{l}\text { Campylopus heterostachys (Hampe) } \\
\text { A. Jaeger }\end{array}$ & $\mathrm{AM}, \mathrm{CA}, \mathrm{CE}, \mathrm{MA}$ & Ampla & Neotropical & Carmo \& Peralta 784 \\
\hline $\begin{array}{l}\text { Campylopus lamellinervis (Müll. Hal.) } \\
\text { Mitt. }\end{array}$ & CA, MA, PM & Moderada & Neotropical & Peralta et al. 9381 \\
\hline Campylopus occultus Mitt. & $\begin{array}{l}\text { AM, CE, MA, PM, } \\
\text { PN }\end{array}$ & Ampla & Neotropical & Carmo \& Peralta 516 \\
\hline Campylopus pilifer Brid. & $\begin{array}{c}\mathrm{AM}, \mathrm{CA}, \mathrm{CE}, \mathrm{MA} \\
\mathrm{PM}\end{array}$ & Ampla & Neotropical & Peralta et al. 9395 \\
\hline Leucobryum albicans (Schwägr.) Lindb. & $\mathrm{AM}, \mathrm{CA}, \mathrm{CE}, \mathrm{MA}$ & Ampla & Neotropical & $\begin{array}{c}\text { Peralta \& Carmo } \\
14835\end{array}$ \\
\hline Leucobryum clavatum Hampe & MA, CE & Moderada & $\begin{array}{l}\text { Endêmica do } \\
\text { Brasil }\end{array}$ & Carmo \& Peralta 520 \\
\hline Leucobryum crispum Müll. Hal. & $\mathrm{AM}, \mathrm{CE}, \mathrm{MA}$ & Ampla & Neotropical & $\begin{array}{c}\text { Peralta \& Gugliota } \\
13558\end{array}$ \\
\hline
\end{tabular}


Tabela 1 (continuação)

\begin{tabular}{l}
\hline Táxons (Divisão/Família/Espécie) \\
\hline Leucobryum giganteum Müll. Hal. \\
Leucomiaceae \\
*Leucomium steerei B.H. Allen \& Veling \\
Leucomium strumosum (Hornsch.) Mitt. \\
Meteoriaceae \\
Floribundaria flaccida (Mitt.) Broth. \\
Meteorium deppei (Hornsch. ex Müll. \\
Hal.) Mitt. \\
Meteorium latifolium (Lindb.) Broth. \\
Meteorium nigrescens (Hedw.) Dozy \\
\& Molk. \\
Meteorium pseudoteres Buck \\
Meteorium teres Mitt. \\
*Toloxis imponderosa (Taylor) Buck \\
Mniaceae \\
Plagiomnium rhynchophorum (Hook.) \\
T.J. Kop.
\end{tabular}

Pohlia tenuifolia (A. Jaeger) Broth.

Neckeraceae

Homalia glabella (Hedw.) Schimp.

Neckera villae-ricae Besch.

Neckeropsis disticha (Hedw.) Kindb.

Neckeropsis undulata (Hedw.) Reichardt

Porotrichum filiferum Mitt.

Porotrichum lancifrons (Hampe) Mitt.

Porotrichum longirostre (Hook.) Mitt.

*Porotrichum mutabile Hampe

Porotrichum substriatum (Hampe) Mitt.

Thamnobryum fasciculatum (Sw. ex Hedw.) I. Sastre

Orthodontiaceae

Hymenodon aeruginosus (Hook. \& Wilson) Müll. Hal.

$\begin{array}{cccc}\text { AM, CE, MA, PN } & \text { Ampla } & \text { América do Sul } & \text { Carmo \& Peralta } 609 \\ \text { CE, MA, PN } & \text { Ampla } & \text { Neotropical } & \text { Carmo \& Peralta } 791 \\ \text { MA } & \text { Moderada } & \text { Endêmica do } & \text { Peralta \& Gugliota } \\ \text { Brasil } & 13731 \\ \text { AM, CE, MA, PN } & \text { Ampla } & \text { Neotropical } & \begin{array}{c}\text { Peralta \& Carmo } \\ 14396\end{array} \\ \text { MA } & \text { Rara } & \text { Neotropical } & \begin{array}{c}\text { Peralta \& Carmo } \\ 14605\end{array} \\ \text { MA } & \text { Rara } & \text { Neotropical } & \begin{array}{c}\text { Peralta \& Gugliota } \\ 13791\end{array} \\ \text { MA } & \text { Rara } & \text { Neotropical } & \begin{array}{c}\text { Peralta \& Gugliota } \\ 13867\end{array}\end{array}$

AM, CE, MA Moderada Cosmopolita Peralta et al. 9312

MA Rara $\quad$ Pantropical $\quad \begin{gathered}\text { Peralta \& Carmo } \\ 14625\end{gathered}$

MA Rara Neotropical

Peralta \& Carmo 14593

Peralta \& Carmo 14284

$\begin{array}{cccc}\text { AM, CE, MA, PN } & \text { Ampla } & \text { Pantropical } & \begin{array}{c}\text { Carmo \& Peralta } 643 \\ \text { Peralta \& Carmo } \\ \text { AM, CA, CE, MA, }\end{array} \\ \text { PN } & \text { Ampla } & \text { Américas } & 14535 \\ \text { CE, MA } & \text { Moderada } & \begin{array}{c}\text { América do Sul } \\ \text { tropical }\end{array} & \begin{array}{c}\text { Peralta \& Gugliota } \\ 13728\end{array} \\ \text { MA } & \text { Moderada } & \text { Neotropical } & \begin{array}{c}\text { Peralta et al. } 9401 \\ \text { Peralta \& Gugliota }\end{array} \\ \text { CE, MA } & \text { Moderada } & \text { Neotropical } & 13493 \\ \text { MA } & \text { Rara } & \text { Neotropical } & \text { Peralta et al. } 9009 \\ \text { AM, MA } & \text { Ampla } & \text { Neotropical e } & \text { Peralta et al. } 8986 \\ & \text { África } & \text { Peralta \& Carmo } \\ \text { MA } & \text { Moderada } & \text { Neotropical } & 14540\end{array}$

MA, PM

Moderada
Neotropical 
Tabela 1 (continuação)

\begin{tabular}{|c|c|c|c|c|}
\hline Táxons (Divisão/Família/Espécie) & $\begin{array}{c}\text { Domínio } \\
\text { Fitogeográfico } \\
\end{array}$ & $\begin{array}{c}\text { Distribuição } \\
\text { Brasil }\end{array}$ & $\begin{array}{c}\text { Distribuição } \\
\text { mundial }\end{array}$ & Voucher \\
\hline \multicolumn{5}{|l|}{ Orthotrichaceae } \\
\hline $\begin{array}{l}\text { Macrocoma orthotrichoides (Raddi) } \\
\text { Wijk. \& Margad. }\end{array}$ & MA & Moderada & Neotropical e Índia & Peralta et al. 9346 \\
\hline Macrocoma tenuis (Hook. \& Grev.) Vitt & CE, MA & Moderada & $\begin{array}{l}\text { Américas, Havaí e } \\
\text { Japão }\end{array}$ & Peralta et al. 8838 \\
\hline Macromitrium cirrosum (Hedw.) Brid. & $\mathrm{AM}, \mathrm{MA}$ & Ampla & Neotropical & Carmo \& Peralta 596 \\
\hline $\begin{array}{l}\text { *Macromitrium longifolium (Hook.) } \\
\text { Brid. }\end{array}$ & MA & Rara & Neotropical & Peralta et al. 8865 \\
\hline $\begin{array}{l}\text { Macromitrium microstomum (Hook. \& } \\
\text { Grev.) Schwägr. }\end{array}$ & MA & Rara & Cosmopolita & Carmo \& Peralta 571 \\
\hline $\begin{array}{l}\text { Macromitrium punctatum (Hook. \& } \\
\text { Grev.) Brid. }\end{array}$ & $\mathrm{AM}, \mathrm{CA}, \mathrm{CE}, \mathrm{MA}$ & Ampla & Neotropical & Carmo \& Peralta 576 \\
\hline Macromitrium richardii Schwägr. & AM, MA & Ampla & $\begin{array}{l}\text { Neotropical e } \\
\text { África }\end{array}$ & Peralta et al. 8992 \\
\hline *Schlotheimia elata Mitt. & MA & Rara & $\begin{array}{l}\text { Endêmica do } \\
\text { Brasil }\end{array}$ & Peralta et al. 9409 \\
\hline Schlotheimia jamesonii (Arn.) Brid. & $\mathrm{AM}, \mathrm{CE}, \mathrm{MA}, \mathrm{PM}$ & Ampla & Neotropical & Peralta et al. 9375 \\
\hline $\begin{array}{l}\text { Schlotheimia rugifolia (Hook.) } \\
\text { Schwägr. }\end{array}$ & $\mathrm{AM}, \mathrm{CE}, \mathrm{MA}$ & Ampla & Neotropical e Índia & Peralta et al. 9356 \\
\hline Schlotheimia tecta Hook. \& Wilson & MA & Moderada & $\begin{array}{l}\text { Neotropical e } \\
\text { México }\end{array}$ & Carmo \& Peralta 534 \\
\hline $\begin{array}{l}\text { Schlotheimia torquata (Sw. ex Hedw.) } \\
\text { Brid. }\end{array}$ & AM, MA & Ampla & Neotropical & Peralta et al. 8823 \\
\hline \multicolumn{5}{|l|}{ Phyllogoniaceae } \\
\hline Phyllogonium viride Brid. & MA & Ampla & $\begin{array}{l}\text { Neotropical e } \\
\text { África }\end{array}$ & $\begin{array}{c}\text { Peralta \& Gugliota } \\
13676\end{array}$ \\
\hline \multicolumn{5}{|l|}{ Pilotrichaceae } \\
\hline $\begin{array}{l}\text { Callicostella merkelii (Hornsch.) A. } \\
\text { Jaeger }\end{array}$ & AM, CE, MA & Ampla & Neotropical & $\begin{array}{c}\text { Peralta \& Gugliota } \\
13741\end{array}$ \\
\hline Callicostella pallida (Hornsch.) Angstr. & $\begin{array}{l}\mathrm{AM}, \mathrm{CA}, \mathrm{CE}, \mathrm{MA} \\
\text { PM, PN }\end{array}$ & Ampla & Neotropical & Carmo \& Peralta 529 \\
\hline $\begin{array}{l}\text { Cyclodictyon limbatum (Hampe) O. } \\
\text { Kuntze }\end{array}$ & MA & Moderada & Neotropical & $\begin{array}{c}\text { Peralta \& Carmo } \\
14759\end{array}$ \\
\hline Cyclodictyon varians (Sull.) Kuntze & $\mathrm{AM}, \mathrm{CE}, \mathrm{MA}$ & Moderada & Américas & $\begin{array}{c}\text { Peralta \& Gugliota } \\
13411\end{array}$ \\
\hline $\begin{array}{l}\text { Hypnella pilifera (Hook. \& Wilson) } \\
\text { A. Jaeger }\end{array}$ & MA & Moderada & Neotropical & $\begin{array}{c}\text { Peralta \& Gugliota } \\
13833\end{array}$ \\
\hline $\begin{array}{l}\text { Lepidopilidium brevisetum (Hampe) } \\
\text { Broth. }\end{array}$ & MA & Moderada & $\begin{array}{l}\text { Endêmica do } \\
\text { Brasil }\end{array}$ & Carmo \& Peralta 656 \\
\hline Lepidopilidium nitens (Hornsch.) Broth. & MA & Moderada & Neotropical & Peralta et al. 9007 \\
\hline Lepidopilum brevipes Mitt. & AM, MA & Moderada & ical & $\begin{array}{c}\text { Peralta \& Gugliota } \\
13582\end{array}$ \\
\hline *Lepidopilum caudicaule Müll. Hal. & MA & Rara & $\begin{array}{l}\text { Endêmica do } \\
\text { Brasil }\end{array}$ & $\begin{array}{c}\text { Peralta \& Gugliota } \\
13410\end{array}$ \\
\hline *Lepidopilum cubense (Sull.) Mitt. & MA & Rara & Neotropical & $\begin{array}{c}\text { Peralta \& Carmo } \\
14191\end{array}$ \\
\hline Lepidopilum longifolium Hampe & MA & Rara & Neotropical & Carmo \& Peralta 525 \\
\hline
\end{tabular}


Tabela 1 (continuação)

\begin{tabular}{|c|c|c|c|c|}
\hline Táxons (Divisão/Família/Espécie) & $\begin{array}{c}\text { Domínio } \\
\text { Fitogeográfico }\end{array}$ & $\begin{array}{l}\text { Distribuição } \\
\text { Brasil }\end{array}$ & $\begin{array}{l}\text { Distribuição } \\
\text { mundial }\end{array}$ & Voucher \\
\hline Lepidopilum muelleri (Hampe) Mitt. & MA & Moderada & Neotropical & $\begin{array}{c}\text { Peralta \& Gugliota } \\
13566\end{array}$ \\
\hline $\begin{array}{l}\text { *Lepidopilum pallidonitens (Müll. Hal.) } \\
\text { Paris }\end{array}$ & $\mathrm{AM}$ & Rara & Neotropical & Carmo \& Peralta 649 \\
\hline $\begin{array}{l}\text { Lepidopilum subsubulatum Geh. \& } \\
\text { Hampe }\end{array}$ & MA & Moderada & $\begin{array}{l}\text { Endêmica do } \\
\text { Brasil }\end{array}$ & $\begin{array}{l}\text { Peralta \& Carmo } \\
14214\end{array}$ \\
\hline Thamniopsis incurva (Hornsch.) Buck & AM, MA & Ampla & Neotropical & Peralta et al. 8856 \\
\hline Thamniopsis langsdorfii (Hook.) Buck & MA & Moderada & Neotropical & Peralta et al. 9026 \\
\hline Thamniopsis undata (Hedw.) Buck & $\mathrm{CE}, \mathrm{MA}$ & Moderada & Neotropical & $\begin{array}{c}\text { Peralta \& Gugliota } \\
13646\end{array}$ \\
\hline $\begin{array}{l}\text { Trachyxiphium guadalupense (Spreng.) } \\
\text { Buck }\end{array}$ & MA & Moderada & Américas & $\begin{array}{c}\text { Peralta \& Gugliota } \\
13501\end{array}$ \\
\hline $\begin{array}{l}\text { Trachyxiphium saxicola (R.S. Willia) } \\
\text { Vaz-Imbassahy \& Costa }\end{array}$ & $\mathrm{MA}, \mathrm{CE}$ & Moderada & Neotropical & $\begin{array}{c}\text { Peralta \& Carmo } \\
14434\end{array}$ \\
\hline $\begin{array}{l}\text { Trachyxiphium variabile (Hornsch. ex } \\
\text { Mitt.) Buck }\end{array}$ & MA & Rara & Neotropical & $\begin{array}{c}\text { Peralta \& Gugliota } \\
13472\end{array}$ \\
\hline \multicolumn{5}{|l|}{ Polytrichaceae } \\
\hline $\begin{array}{l}\text { Atrichum androgynum (Müll. Hal.) A. } \\
\text { Jaeger }\end{array}$ & MA & Moderada & $\begin{array}{l}\text { Neotropical e } \\
\quad \text { África }\end{array}$ & $\begin{array}{c}\text { Peralta \& Carmo } \\
14828\end{array}$ \\
\hline $\begin{array}{l}\text { Pogonatum campylocarpum (Müll. } \\
\text { Hal.) Mitt. }\end{array}$ & MA & Moderada & Neotropical & Carmo \& Peralta 764 \\
\hline Polytrichum commune L. ex Hedw. & $\mathrm{AM}, \mathrm{CE}, \mathrm{MA}$ & Ampla & Cosmopolita & $\begin{array}{c}\text { Peralta \& Gugliota } \\
13794\end{array}$ \\
\hline $\begin{array}{l}\text { Polyrichum juniperinum Wild. ex } \\
\text { Hedw. }\end{array}$ & $\mathrm{AM}, \mathrm{CE}, \mathrm{MA}$ & Ampla & Cosmopolita & Peralta et al. 9368 \\
\hline \multicolumn{5}{|l|}{ Pottiaceae } \\
\hline Barbula arcuata Griff. & $\mathrm{AM}, \mathrm{CE}, \mathrm{MA}$ & Moderada & Cosmopolita & $\begin{array}{c}\text { Peralta \& Carmo } \\
14636\end{array}$ \\
\hline Barbula indica (Hook.) Spreng. & $\begin{array}{l}\mathrm{AM}, \mathrm{CA}, \mathrm{CE}, \mathrm{MA}, \\
\text { PN }\end{array}$ & Ampla & Pantropical & Carmo \& Peralta 720 \\
\hline Streptopogon calymperes Müll. Hal. & MA & Rara & Neotropical & Peralta et al. 9340 \\
\hline Tortella humilis (Hedw.) Jenn. & $\begin{array}{l}\text { CA, CE, MA, PM, } \\
\text { PN }\end{array}$ & Ampla & Cosmopolita & $\begin{array}{c}\text { Peralta \& Gugliota } \\
13551\end{array}$ \\
\hline \multicolumn{5}{|l|}{ Prionodontaceae } \\
\hline $\begin{array}{l}\text { Prionodon densus (Sw. ex Hedw.) } \\
\text { Müll. Hal. }\end{array}$ & MA, PM & Moderada & Pantropical & Peralta et al. 9010 \\
\hline \multicolumn{5}{|l|}{ Pterobryaceae } \\
\hline *Orthostichopsis latifolia Sehnem & MA & Rara & $\begin{array}{l}\text { Endêmica do } \\
\text { Brasil }\end{array}$ & $\begin{array}{c}\text { Peralta \& Gugliota } \\
13861\end{array}$ \\
\hline $\begin{array}{l}\text { Orthostichopsis tortipilis (Müll. Hal.) } \\
\text { Broth. }\end{array}$ & AM, MA & Moderada & Neotropical & Carmo \& Peralta 780 \\
\hline Pterobryon densum Hornsch. & MA & Moderada & Neotropical & $\begin{array}{c}\text { Peralta \& Gugliota } \\
13470\end{array}$ \\
\hline $\begin{array}{l}\text { Spiredentopsis longissima (Raddi) } \\
\text { Broth. }\end{array}$ & MA & Moderada & $\begin{array}{l}\text { América do Sul } \\
\text { tropical }\end{array}$ & $\begin{array}{l}\text { Peralta \& Carmo } \\
14834\end{array}$ \\
\hline
\end{tabular}


Tabela 1 (continuação)

\begin{tabular}{|c|c|c|c|c|}
\hline Táxons (Divisão/Família/Espécie) & $\begin{array}{l}\text { Domínio } \\
\text { Fitogeográfico }\end{array}$ & $\begin{array}{l}\text { Distribuição } \\
\text { Brasil }\end{array}$ & $\begin{array}{l}\text { Distribuição } \\
\text { mundial }\end{array}$ & Voucher \\
\hline \multicolumn{5}{|l|}{ Ptychomitriaceae } \\
\hline Ptychomitrium vaginatum Besch. & MA, PM & Moderada & Brasil e Paraguai & Peralta et al. 9414 \\
\hline \multicolumn{5}{|l|}{ Pylaisiadelphaceae } \\
\hline Isopterygium affusum Mitt. & $\mathrm{AM}, \mathrm{CE}, \mathrm{MA}$ & Moderada & Neotropical & $\begin{array}{c}\text { Peralta \& Gugliota } \\
13494\end{array}$ \\
\hline $\begin{array}{l}\text { Isopterygium subbrevisetum (Hampe) } \\
\text { Broth. }\end{array}$ & AM, MA & Ampla & Neotropical & $\begin{array}{c}\text { Peralta \& Gugliota } \\
13640\end{array}$ \\
\hline Isopterygium tenerifolium Mitt. & $\mathrm{AM}, \mathrm{CE}, \mathrm{MA}$ & Ampla & Neotropical & Peralta et al. 9404 \\
\hline Pylaisiadelpha brasiliensis H.A. Crum & MA & Rara & $\begin{array}{l}\text { Endêmica do } \\
\text { Brasil }\end{array}$ & $\begin{array}{c}\text { Peralta \& Carmo } \\
14228\end{array}$ \\
\hline Wijkia flagellifera (Broth.) H.A. Crum & MA & Moderada & Neotropical & Peralta et al. 8958 \\
\hline \multicolumn{5}{|l|}{ Racopilaceae } \\
\hline Racopilum tomentosum (Hedw.) Brid. & $\begin{array}{l}\mathrm{AM}, \mathrm{CE}, \mathrm{MA}, \mathrm{PM} \\
\text { PN }\end{array}$ & Ampla & Cosmopolita & Peralta et al. 9042 \\
\hline \multicolumn{5}{|l|}{ Rhacocarpaceae } \\
\hline $\begin{array}{l}\text { Rhacocarpus purpurascens (Brid.) } \\
\text { Müll. Hal. }\end{array}$ & MA & Moderada & Cosmopolita & Peralta et al. 9285 \\
\hline \multicolumn{5}{|l|}{ Rhizogoniaceae } \\
\hline $\begin{array}{l}\text { Pyrrhobryum spiniforme (Hedw.) Mitt. } \\
\text { Sematophyllaceae }\end{array}$ & \multicolumn{3}{|c|}{ Sematophyllaceae } & Carmo \& Peralta 684 \\
\hline $\begin{array}{l}\text { Acroporium estrellae (Müll. Hal.) Buck } \\
\text { \& Schäf.-Verw. }\end{array}$ & $\mathrm{CE}, \mathrm{MA}$ & Ampla & Neotropical & Carmo \& Peralta 564 \\
\hline $\begin{array}{l}\text { Acroporium exiggum (Broth.) Buck \& } \\
\text { Schäf.-Verw. }\end{array}$ & MA & Rara & $\begin{array}{l}\text { Endêmica do } \\
\text { Brasil }\end{array}$ & $\begin{array}{c}\text { Peralta \& Carmo } \\
14186\end{array}$ \\
\hline Acroporium longirostre (Brid.) Buck & $\mathrm{AM}, \mathrm{CE}, \mathrm{MA}$ & Ampla & Neotropical & $\begin{array}{c}\text { Peralta \& Carmo } \\
14514\end{array}$ \\
\hline Acroporium pungens (Hedw.) Broth. & $\mathrm{AM}, \mathrm{CE}, \mathrm{MA}$ & Moderada & Neotropical & Peralta et al. 8894 \\
\hline Aptychopsis pungifolia (Hampe) Borth. & MA & Moderada & $\begin{array}{l}\text { Endêmica do } \\
\text { Brasil }\end{array}$ & Peralta et al. 9348 \\
\hline Donnellia commutata (Müll. Hal.) Buck & $\mathrm{AM}, \mathrm{CE}, \mathrm{MA}, \mathrm{PN}$ & Moderada & Neotropical & $\begin{array}{c}\text { Peralta \& Carmo } \\
14375\end{array}$ \\
\hline $\begin{array}{l}\text { Paranapiacabaea paulista Buck \& } \\
\text { Vital }\end{array}$ & MA & Rara & $\begin{array}{l}\text { Endêmica do } \\
\text { Brasil }\end{array}$ & Carmo \& Peralta 603 \\
\hline $\begin{array}{l}\text { Sematophyllum beyrichii (Hornsch.) } \\
\text { Broth. }\end{array}$ & MA & Moderada & Neotropical & $\begin{array}{c}\text { Peralta \& Gugliota } \\
13591\end{array}$ \\
\hline Sematophyllum cuspidiferum Mitt. & $\mathrm{CE}, \mathrm{MA}, \mathrm{PN}$ & Ampla & Neotropical & Peralta et al. 8811 \\
\hline $\begin{array}{l}\text { Sematophyllum galipense (Müll. Hal.) } \\
\text { Mitt. }\end{array}$ & $\mathrm{AM}, \mathrm{CE}, \mathrm{MA}$ & Ampla & Neotropical & $\begin{array}{c}\text { Peralta \& Gugliota } \\
13824\end{array}$ \\
\hline $\begin{array}{l}\text { *Sematophyllum litophilum (Hornsch.) } \\
\text { Ångstr. }\end{array}$ & $\mathrm{AM}, \mathrm{CE}, \mathrm{MA}$ & Rara & Neotropical & $\begin{array}{c}\text { Peralta \& Gugliota } \\
13837\end{array}$ \\
\hline $\begin{array}{l}\text { Sematophyllum subpinnatum (Brid.) } \\
\text { Britt. }\end{array}$ & $\begin{array}{l}\mathrm{AM}, \mathrm{CA}, \mathrm{CE}, \mathrm{MA} \\
\text { PM, PN }\end{array}$ & Ampla & Pantropical & Peralta et al. 8903 \\
\hline $\begin{array}{l}\text { Sematophyllum subsimplex (Hedw.) } \\
\text { Mitt. }\end{array}$ & $\begin{array}{c}\mathrm{AM}, \mathrm{CA}, \mathrm{CE}, \mathrm{MA} \\
\text { PN }\end{array}$ & Ampla & Neotropical & Carmo \& Peralta 626 \\
\hline $\begin{array}{l}\text { Sematophyllum swartzii (Schwägr.) } \\
\text { Welch \& H.A. Crum }\end{array}$ & MA & Moderada & Neotropical & Peralta et al. 8915 \\
\hline
\end{tabular}


Tabela 1 (continuação)

\begin{tabular}{|c|c|c|c|c|}
\hline Táxons (Divisão/Família/Espécie) & $\begin{array}{c}\text { Domínio } \\
\text { Fitogeográfico }\end{array}$ & $\begin{array}{l}\text { Distribuição } \\
\text { Brasil }\end{array}$ & $\begin{array}{l}\text { Distribuição } \\
\text { mundial }\end{array}$ & Voucher \\
\hline Trichosteleum glaziovii (Hampe) Buck & MA & Moderada & $\begin{array}{l}\text { Endêmica do } \\
\text { Brasil }\end{array}$ & $\begin{array}{c}\text { Peralta \& Gugliota } \\
13886\end{array}$ \\
\hline \multicolumn{5}{|l|}{ Sphagnaceae } \\
\hline Sphagnum aciphyllum Müll. Hal. & MA, PM & Moderada & $\begin{array}{l}\text { América do Sul } \\
\text { tropical }\end{array}$ & Carmo \& Peralta 75 \\
\hline Sphagnum platyphylloides Warnst. & $\mathrm{MA}, \mathrm{CE}$ & Rara & $\begin{array}{l}\text { Endêmica do } \\
\text { Brasil }\end{array}$ & $\begin{array}{c}\text { Peralta \& Gugliota } \\
13834\end{array}$ \\
\hline \multicolumn{5}{|l|}{ Thuidiaceae } \\
\hline Pelekium scabrosulum (Mitt.) Touw & $\mathrm{AM}, \mathrm{CE}, \mathrm{MA}$ & Ampla & Neotropical & $\begin{array}{c}\text { Peralta \& Carmo } \\
14646\end{array}$ \\
\hline $\begin{array}{l}\text { Thuidiopsis sparsa (Hook. \& Wilson) } \\
\text { Broth. }\end{array}$ & MA & Moderada & Neotropical & $\begin{array}{c}\text { Peralta \& Gugliota } \\
13505\end{array}$ \\
\hline $\begin{array}{l}\text { Thuidium pseudoprotensum (Müll. } \\
\text { Hal.) Mitt. }\end{array}$ & MA & Moderada & Neotropical & $\begin{array}{c}\text { Peralta \& Carmo } \\
14653\end{array}$ \\
\hline $\begin{array}{l}\text { Thuidium tamariscinum (Hedw.) Bruch } \\
\text { \& Schimp. }\end{array}$ & MA, PM & Moderada & Cosmopolita & Peralta \& Carmo \\
\hline Thuidium tomentosum Schimp. & $\mathrm{AM}, \mathrm{CE}, \mathrm{MA}, \mathrm{PN}$ & Ampla & Neotropical & $\begin{array}{c}\text { Peralta \& Gugliota } \\
13788\end{array}$ \\
\hline
\end{tabular}

Marchantiophyta

Acrobolbaceae

Tylimanthus laxus (Lehm. \& Lindenb.) Steph.

CE, MA, PN Moderada América tropical

Peralta et al. 9391

Aneuraceae

Aneura pinguis (L.) Dumot.

Riccardia amazonica (Spruce) Schiffn. ex Gradst. \& Hekking

Riccardia chamedryfolia (With.) Grolle

Riccardia digitiloba (Spruce ex Steph.) Pagán

$\begin{array}{cccc}\text { AM, MA, PN } & \text { Ampla } & \text { Subcosmopolita } & \text { Peralta et al. } 9371 \\ \text { AM, CE, MA } & \text { Ampla } & \text { América tropical } & \text { Carmo \& Peralta } 705 \\ \text { CE, MA } & \text { Ampla } & \text { Neotropical } & \text { Peralta \& Carmo } \\ & & & 14621 \\ \text { Ampla } & \text { América tropical } & \begin{array}{c}\text { Peralta \& Gugliota } \\ 13579\end{array} \\ \text { MA } & \text { Rara } & \text { América tropical } & \text { Peralta \& Carmo } \\ & & & 14149\end{array}$

Riccardia glaziovii (Spruce) Meenks

Riccardia metzgeriiformis (Steph.) Schiffn.

$\begin{array}{cc}\text { AM, MA } & \text { Moderada } \\ \text { MA } & \text { Moderada } \\ \text { MA, CE } & \text { Ampla }\end{array}$

Neotropical

Peralta et al. 8816

América do Sul Peralta et al. s.n. (SP tropical 420887)

Riccardia regnellii (Angstr.) Hell

Riccardia tenuicola (Spruce) Schiffn. ex Meenks

AM, MA Moderada

Endêmica do Brasil

Carmo \& Peralta 687

Balantiopsaceae

Isotachis aubertii (Schwägr.) Mitt.

Isotachis serrulata (Sw.) Gottsche

MA

AM, MA

MA, CE
Moderada

Moderada

Moderada
América do Sul e África América tropical Andes tropical e Brasil
Peralta et al. 8890

Peralta et al. 9306

Peralta \& Gugliota

13831 
Tabela 1 (continuação)

\begin{tabular}{|c|c|c|c|c|}
\hline Táxons (Divisão/Família/Espécie) & $\begin{array}{c}\text { Domínio } \\
\text { Fitogeográfico }\end{array}$ & $\begin{array}{l}\text { Distribuição } \\
\text { Brasil }\end{array}$ & $\begin{array}{l}\text { Distribuição } \\
\text { mundial }\end{array}$ & Voucher \\
\hline \multicolumn{5}{|l|}{ Calypogeiaceae } \\
\hline Calypogeia laxa Gottsche \& Lindenb. & AM, MA & Ampla & América tropical & $\begin{array}{c}\text { Peralta \& Gugliota } \\
13488\end{array}$ \\
\hline Calypogeia peruviana Nees \& Mont. & $\mathrm{AM}, \mathrm{CE}, \mathrm{MA}$ & Ampla & América tropical & $\begin{array}{c}\text { Peralta \& Carmo } \\
14489\end{array}$ \\
\hline \multicolumn{5}{|l|}{ Cephaloziaceae } \\
\hline $\begin{array}{l}\text { Odontoschisma denudatum (Nees) } \\
\text { Dumort. }\end{array}$ & $\mathrm{AM}, \mathrm{CE}, \mathrm{MA}$ & Moderada & Cosmopolita & Peralta et al. 9308 \\
\hline Odontoschisma falcifolium Steph. & $\mathrm{AM}, \mathrm{CE}, \mathrm{MA}$ & Moderada & $\begin{array}{l}\text { América do Sul } \\
\text { tropical }\end{array}$ & $\begin{array}{c}\text { Peralta \& Carmo } \\
14588\end{array}$ \\
\hline $\begin{array}{l}\text { Odontoschisma longiflorum (Taylor) } \\
\text { Steph. }\end{array}$ & MA, CE & Moderada & América tropical & $\begin{array}{c}\text { Peralta \& Carmo } \\
14735\end{array}$ \\
\hline $\begin{array}{l}\text { Cylindrocolea rhizantha (Mont.) } \\
\text { Schust. }\end{array}$ & $\mathrm{AM}, \mathrm{CE}, \mathrm{MA}$ & Moderada & Neotropical & $\begin{array}{c}\text { Peralta \& Gugliota } \\
13522\end{array}$ \\
\hline $\begin{array}{l}\text { Kymatocalyx dominicensis (Spruce) } \\
\text { Váňa }\end{array}$ & MA & Moderada & $\begin{array}{l}\text { América do Sul e } \\
\text { África }\end{array}$ & $\begin{array}{c}\text { Peralta \& Gugliota } \\
13820\end{array}$ \\
\hline \multicolumn{5}{|l|}{ Frullaniaceae } \\
\hline $\begin{array}{l}\text { Frullania apiculata (Reinw. et al.) } \\
\text { Dumort. }\end{array}$ & $\mathrm{AM}, \mathrm{CE}, \mathrm{MA}$ & Ampla & Pantropical & Carmo \& Peralta 727 \\
\hline Frullania atrata (Sw.) Nees & AM, MA & Ampla & América tropical & Carmo \& Peralta 551 \\
\hline Frullania brasiliensis Raddi & $\mathrm{MA}, \mathrm{CE}$ & Ampla & América tropical & $\begin{array}{c}\text { Peralta \& Carmo } \\
14377\end{array}$ \\
\hline Frullania breuteliana Gottsche & MA & Moderada & Neotropical & $\begin{array}{c}\text { Peralta \& Carmo } \\
14478\end{array}$ \\
\hline Frullania caulisequa (Nees) Nees & $\begin{array}{l}\mathrm{AM}, \mathrm{CA}, \mathrm{CE}, \mathrm{MA}, \\
\text { PM }\end{array}$ & Ampla & América tropical & $\begin{array}{c}\text { Peralta \& Carmo } \\
14357\end{array}$ \\
\hline $\begin{array}{l}\text { Frullania curvilobula Schäf.-Verw. } \\
\text { et al. }\end{array}$ & MA & Rara & $\begin{array}{l}\text { Endêmica do } \\
\text { Brasil }\end{array}$ & $\begin{array}{c}\text { Peralta \& Gugliota } \\
13852\end{array}$ \\
\hline Frullania ericoides (Nees) Mont. & $\begin{array}{l}\text { AM, CA, CE, MA, } \\
\text { PM, PN }\end{array}$ & Ampla & Pantropical & Peralta et al. 8946 \\
\hline Frullania flexicaulis Spruce & MA & Rara & América tropical & $\begin{array}{c}\text { Peralta \& Gugliota } \\
13523\end{array}$ \\
\hline Frullania grossifolia Steph. & MA & Rara & Neotropical & $\begin{array}{c}\text { Peralta \& Gugliota } \\
13554\end{array}$ \\
\hline Frullania involuta Hampe ex Steph. & MA & Rara & Neotropical & Peralta et al. 9386 \\
\hline $\begin{array}{l}\text { Frullania kunzei (Lehm. \& Lindenb.) } \\
\text { Lehm. \& Lindenb. }\end{array}$ & $\mathrm{AM}, \mathrm{CE}, \mathrm{MA}, \mathrm{PN}$ & Ampla & América tropical & Peralta et al. 8853 \\
\hline Frullania obscura Stephani & $\mathrm{AM}, \mathrm{CE}, \mathrm{MA}$ & Ampla & Pantropical & Peralta et al. 8938 \\
\hline Frullania setigera Steph. & MA & Moderada & América tropical & $\begin{array}{c}\text { Peralta \& Gugliota } \\
13513\end{array}$ \\
\hline Frullania vitalii Yuzawa \& Hatt. & CE, MA & Moderada & $\begin{array}{l}\text { Endêmica do } \\
\text { Brasil }\end{array}$ & $\begin{array}{c}\text { Peralta \& Carmo } \\
14438\end{array}$ \\
\hline \multicolumn{5}{|l|}{ Geocalycaceae } \\
\hline $\begin{array}{l}\text { Saccogynidium caldense (Angstr.) } \\
\text { Grolle }\end{array}$ & MA & Moderada & $\begin{array}{l}\text { Endêmica do } \\
\text { Brasil }\end{array}$ & Peralta et al. 9382 \\
\hline
\end{tabular}


Tabela 1 (continuação)

\begin{tabular}{l}
\hline Táxons (Divisão/Família/Espécie) \\
\hline Herbetaceae \\
Herbertus bivittatus Spruce \\
Jamesoniellaceae \\
Jamesoniella rubricaulis(Nees) Grolle \\
Syzygyella anomala (Lindenb. \& \\
Gottsche) Stephani \\
Syzygyella liberata Inoue \\
Syzygyella perfoliata (Sw.) Spruce
\end{tabular}

Jungermanniaceae

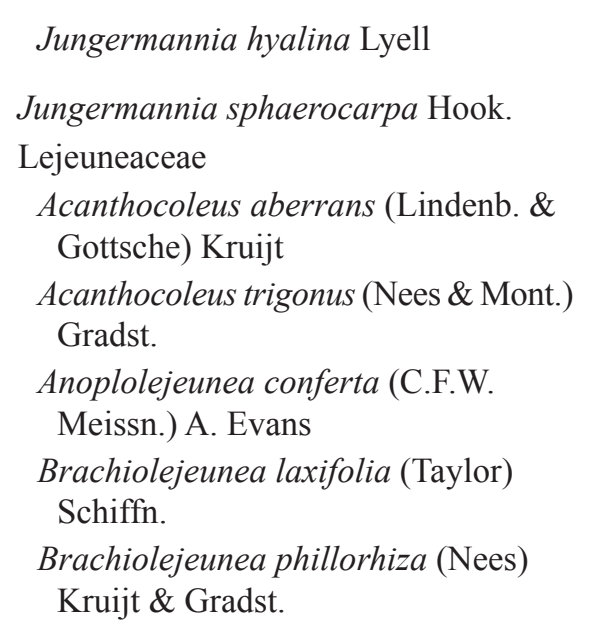

\begin{tabular}{cccc}
$\begin{array}{c}\text { Domínio } \\
\text { Fitogeográfico }\end{array}$ & $\begin{array}{c}\text { Distribuição } \\
\text { Brasil }\end{array}$ & $\begin{array}{c}\text { Distribuição } \\
\text { mundial }\end{array}$ & Voucher \\
\hline
\end{tabular}

AM, MA Moderada América tropical Peralta et al. 8993

$\begin{array}{cccc}\text { MA } & \text { Moderada } & \text { Neotropical } & 13735 \\ \text { MA } & \text { Rara } & \text { Neotropical } & \begin{array}{c}\text { Peralta \& Gugliota } \\ 13733\end{array} \\ \text { MA } & \text { Moderada } & \text { Neotropical } & \begin{array}{c}\text { Peralta \& Carmo } \\ 14644\end{array} \\ & & & \text { Peralta \& Carmo } \\ \text { MA } & \text { Moderada } & \text { América tropical } & 14400\end{array}$

$\begin{array}{cccc}\text { CE, MA, PN } & \text { Moderada } & \text { Cosmopolita } & \text { Peralta \& Gugliota } \\ \text { MA } & \text { Rara } & \text { Cosmopolita } & \text { Peralta et al. } 9358\end{array}$

\begin{tabular}{|c|c|c|}
\hline CE, MA, PM & Ampla & $\begin{array}{c}\text { América tropical e } \\
\text { África }\end{array}$ \\
\hline MA & Moderada & Neotropical \\
\hline $\mathrm{AM}, \mathrm{MA}$ & Ampla & América tropical \\
\hline MA & Moderada & América tropical \\
\hline $\mathrm{AM}, \mathrm{MA}$ & Moderada & $\begin{array}{c}\text { América tropical e } \\
\text { África }\end{array}$ \\
\hline
\end{tabular}
Bryopteris diffusa (Sw.) Nees
Bryopteris filicina (Sw.) Nees

Ceratolejeunea coarina(Gottsche) Steph.

$\begin{array}{cccc}\text { AM, CE, MA } & \text { Ampla } & \text { Neotropical } & \begin{array}{c}\text { Peralta \& Carmo } \\ 14239\end{array} \\ \text { AM, CE, MA, PN } & \text { Ampla } & \text { Neotropical } & \begin{array}{c}\text { Carmo \& Peralta } 608 \\ \text { Peralta \& Carmo } \\ 14247\end{array} \\ \text { AM, MA } & \text { Moderada } & \text { América tropical } & \begin{array}{c}\text { Peralta \& Gugliota } \\ \text { AM, MA }\end{array} \\ \text { Ampla } & \text { América tropical } & 13586 \\ \text { AM, MA } & \text { Ampla } & \text { América tropical } & \begin{array}{c}\text { Peralta \& Carmo } \\ 14168\end{array}\end{array}$

Ceratolejeunea cornuta (Lindenb.) Schiffn.

Ceratolejeunea cubensis(Mont.)

Schiffn.

Ceratolejeunea fallax (Lehm. \& Lindenb.) Bonner

AM, MA Moderada América tropical

Peralta \& Carmo 14219

Peralta et al. 8942

Peralta \& Carmo 14408

Peralta \& Carmo 14376

Peralta \& Carmo 14365

Cheilolejeunea acutangula (Nees) Grolle

Cheilolejeunea beyrichii (Lindenb.)

Reiner

$\begin{array}{cccc}\text { AM, CE, MA } & \text { Ampla } & \text { Neotropical } & \text { Peralta et al. } 8919 \\ \text { CA, CE } & \text { Rara } & \text { Endêmica do } & \text { Peralta \& Gugliota } \\ & & \text { Brasil } & 13729 \\ \text { AM, MA } & \text { Rara } & \text { América do Sul } & \text { Peralta \& Gugliota } \\ & \text { tropical } & 13737 \\ \text { CE, MA, PN } & \text { Moderada } & \text { América tropical } & \text { Peralta \& Gugliota } \\ & & & 13821\end{array}$

Cheilolejeunea comans (Spruce)

Schust.

Cheilolejeunea discoidea (Lehm. \& Lindenb.) Kachr. \& Schust. 
Tabela 1 (continuação)

\begin{tabular}{|c|c|c|c|c|}
\hline Táxons (Divisão/Família/Espécie) & $\begin{array}{c}\text { Domínio } \\
\text { Fitogeográfico }\end{array}$ & $\begin{array}{l}\text { Distribuição } \\
\text { Brasil }\end{array}$ & $\begin{array}{c}\text { Distribuição } \\
\text { mundial }\end{array}$ & Voucher \\
\hline $\begin{array}{l}\text { Cheilolejeunea filiformis (Sw.) W. Ye, } \\
\text { R.L. Zhu \& Gradst. }\end{array}$ & $\mathrm{AM}, \mathrm{MA}, \mathrm{PN}$ & Ampla & América tropical & Carmo \& Peralta 517 \\
\hline $\begin{array}{l}\text { Cheilolejeunea holostipa (Spruce) } \\
\text { Grolle \& R.L. Zhu }\end{array}$ & AM, MA & Moderada & América tropical & $\begin{array}{c}\text { Peralta \& Gugliota } \\
13704\end{array}$ \\
\hline $\begin{array}{l}\text { Cheilolejeunea trifaria (Reinw. et al.) } \\
\text { Mizut. }\end{array}$ & $\mathrm{AM}, \mathrm{CE}, \mathrm{MA}, \mathrm{PN}$ & Ampla & Pantropical & $\begin{array}{c}\text { Peralta \& Carmo } \\
14556\end{array}$ \\
\hline $\begin{array}{l}\text { Cheilolejeunea unciloba (Lindenb.) } \\
\text { Malombe }\end{array}$ & $\mathrm{MA}, \mathrm{CE}$ & Moderada & $\begin{array}{c}\text { América tropical e } \\
\text { África }\end{array}$ & $\begin{array}{l}\text { Peralta \& Carmo } \\
14195\end{array}$ \\
\hline $\begin{array}{l}\text { Cheilolejeunea xanthocarpa (Lehm. \& } \\
\text { Lindenb.) Malombe }\end{array}$ & MA, CE & Moderada & Pantropical & $\begin{array}{c}\text { Peralta \& Carmo } \\
14380\end{array}$ \\
\hline $\begin{array}{l}\text { Cololejeunea cardiocarpa (Mont.) A. } \\
\text { Evans }\end{array}$ & $\mathrm{AM}, \mathrm{CE}, \mathrm{MA}$ & Ampla & Pantropical & $\begin{array}{c}\text { Peralta \& Gugliota } \\
13739\end{array}$ \\
\hline Cololejeunea clavatopapillata Steph. & MA & Rara & América tropical & Peralta et al. 8905 \\
\hline Cololejeunea gracilis (Jovet-Ast.) Pócs. & $\mathrm{AM}, \mathrm{CE}, \mathrm{MA}$ & Moderada & Neotropical & $\begin{array}{c}\text { Peralta \& Carmo } \\
14543\end{array}$ \\
\hline $\begin{array}{l}\text { Cololejeunea manaosensis(Herzog) } \\
\text { O. Yano }\end{array}$ & AM, MA & Rara & Neotropical & $\begin{array}{c}\text { Peralta \& Carmo } \\
14165\end{array}$ \\
\hline $\begin{array}{l}\text { Cololejeunea microscopica (Taylor) } \\
\text { Schiffn. }\end{array}$ & MA & Rara & $\begin{array}{c}\text { América tropical e } \\
\text { África }\end{array}$ & Peralta et al. 8822 \\
\hline $\begin{array}{l}\text { Cololejeunea minutissima }(\mathrm{Sm} .) \\
\text { Schiffn. }\end{array}$ & $\mathrm{AM}, \mathrm{CE}, \mathrm{MA}, \mathrm{PN}$ & Ampla & Pantropical & $\begin{array}{c}\text { Peralta \& Carmo } \\
14568\end{array}$ \\
\hline $\begin{array}{l}\text { Cololejeunea papilliloba (Steph.) } \\
\text { Steph. }\end{array}$ & MA, PM & Rara & Neotropical & $\begin{array}{c}\text { Peralta \& Gugliota } \\
13804\end{array}$ \\
\hline $\begin{array}{l}\text { Cololejeunea paucifolia (Spruce) } \\
\text { Bernecker \& Pócs }\end{array}$ & MA & Rara & Neotropical & $\begin{array}{c}\text { Peralta \& Carmo } \\
14333\end{array}$ \\
\hline $\begin{array}{l}\text { Cololejeunea truncatifolia (Horik.) } \\
\text { Mizut. }\end{array}$ & $\mathrm{AM}, \mathrm{CE}, \mathrm{MA}$ & Ampla & Pantropical & $\begin{array}{l}\text { Peralta \& Carmo } \\
14190\end{array}$ \\
\hline *Colura calyptrifolia (Hook.) Dumort. & MA & Rara & América tropical & $\begin{array}{c}\text { Peralta \& Carmo } \\
14266\end{array}$ \\
\hline Colura greig-smithii Jovet-Ast & $\mathrm{AM}, \mathrm{CE}, \mathrm{MA}$ & Rara & $\begin{array}{l}\text { América do Sul } \\
\text { tropical }\end{array}$ & $\begin{array}{c}\text { Peralta \& Carmo } \\
14175\end{array}$ \\
\hline Colura tenuicornis(A. Evans) Steph. & MA & Moderada & Pantropical & Carmo \& Peralta 777 \\
\hline Cyclolejeunea luteola (Spruce) Grolle & $\mathrm{AM}, \mathrm{CE}, \mathrm{MA}$ & Moderada & América tropical & $\begin{array}{c}\text { Peralta \& Gugliota } \\
13537\end{array}$ \\
\hline $\begin{array}{l}\text { Dicranolejeunea axilaris (Nees \& } \\
\text { Mont.) Schiffn. }\end{array}$ & MA & Rara & Neotropical & $\begin{array}{l}\text { Peralta \& Carmo } \\
14703\end{array}$ \\
\hline Diplasiolejeunea brunnea Steph. & $\mathrm{AM}, \mathrm{CE}, \mathrm{MA}$ & Ampla & América tropical & $\begin{array}{c}\text { Peralta \& Gugliota } \\
13601\end{array}$ \\
\hline Diplasiolejeunea cavifolia Steph. & MA & Moderada & Pantropical & $\begin{array}{c}\text { Peralta \& Carmo } \\
14471\end{array}$ \\
\hline $\begin{array}{l}\text { Drepanolejeunea anoplantha (Spruce) } \\
\text { Steph. }\end{array}$ & AM, MA & Moderada & Neotropical & $\begin{array}{c}\text { Peralta \& Carmo } \\
14820\end{array}$ \\
\hline Drepanolejeunea araucariae Steph. & MA & Moderada & Neotropical & $\begin{array}{c}\text { Peralta \& Carmo } \\
14757\end{array}$ \\
\hline Drepanolejeunea fragilis Bischl. & AM, MA & Ampla & América tropical & $\begin{array}{c}\text { Peralta \& Gugliota } \\
13649\end{array}$ \\
\hline
\end{tabular}


Tabela 1 (continuação)

\begin{tabular}{|c|c|c|c|c|}
\hline Táxons (Divisão/Família/Espécie) & $\begin{array}{c}\text { Domínio } \\
\text { Fitogeográfico }\end{array}$ & $\begin{array}{l}\text { Distribuição } \\
\text { Brasil }\end{array}$ & $\begin{array}{l}\text { Distribuição } \\
\text { mundial }\end{array}$ & Voucher \\
\hline $\begin{array}{l}\text { Drepanolejeunea granatensis (J.B. Jack } \\
\text { \& Steph.) Bischl. }\end{array}$ & MA & Rara & Neotropical & Peralta et al. 8821 \\
\hline $\begin{array}{l}\text { Drepanolejeunea lichenicola (Sruce) } \\
\text { Steph. }\end{array}$ & MA & Rara & América tropical & $\begin{array}{c}\text { Peralta \& Carmo } \\
14564\end{array}$ \\
\hline Drepanolejeunea mosenii Bischl. & AM, MA & Ampla & América tropical & $\begin{array}{c}\text { Peralta \& Gugliota } \\
13528\end{array}$ \\
\hline Drepanolejeunea orthophylla Bischl. & $\mathrm{AM}, \mathrm{MA}$ & Moderada & América tropical & $\begin{array}{c}\text { Peralta \& Carmo } \\
14297\end{array}$ \\
\hline Frullanoides densifolia Raddi & $\mathrm{AM}, \mathrm{CE}, \mathrm{MA}$ & Moderada & América tropical & Peralta et al. 8994 \\
\hline Frullanoides tristis (Steph.) Slageren & MA & Moderada & $\begin{array}{c}\text { América tropical e } \\
\text { África }\end{array}$ & Peralta et al. 8953 \\
\hline $\begin{array}{l}\text { Harpalejeunea oxyphylla (Nees \& } \\
\text { Mont.) Steph. }\end{array}$ & AM, MA & Moderada & América tropical & $\begin{array}{c}\text { Peralta \& Gugliota } \\
13507\end{array}$ \\
\hline $\begin{array}{l}\text { Harpalejeunea stricta (Lindenb. \& } \\
\text { Gottsche) Steph. }\end{array}$ & AM, MA & Moderada & América tropical & Peralta et al. 8839 \\
\hline $\begin{array}{l}\text { Harpalejeunea tridens (Besch. \& } \\
\text { Spruce) Steph. }\end{array}$ & MA & Rara & Neotropical & $\begin{array}{c}\text { Peralta \& Carmo } \\
14391\end{array}$ \\
\hline Lejeunea aphanes Spruce & MA, PN & Rara & Neotropical & $\begin{array}{c}\text { Peralta \& Gugliota } \\
13560\end{array}$ \\
\hline $\begin{array}{l}\text { Lejeunea bermudiana (A. Evans) R.M. } \\
\text { Schust. }\end{array}$ & AM, MA & Moderada & $\begin{array}{c}\text { América tropical e } \\
\text { África }\end{array}$ & $\begin{array}{c}\text { Peralta \& Gugliota } \\
13424\end{array}$ \\
\hline Lejeunea capensis Gottsche & MA & Rara & Pantropical & $\begin{array}{c}\text { Peralta \& Carmo } \\
14483\end{array}$ \\
\hline $\begin{array}{l}\text { Lejeunea caulicalyx (Steph.) E. Reiner } \\
\text { \& Goda }\end{array}$ & AM, CE, MA, PN & Ampla & América tropical & $\begin{array}{c}\text { Peralta \& Carmo } \\
14285\end{array}$ \\
\hline Lejeunea controversa Gottsche & $\mathrm{AM}, \mathrm{MA}, \mathrm{PN}$ & Moderada & América tropical & Peralta et al. 9351 \\
\hline $\begin{array}{l}\text { Lejeunea cristulaeflora (Gottsche ex } \\
\text { Steph.) M. E. Reiner \& Goda }\end{array}$ & MA & Moderada & $\begin{array}{c}\text { Endêmica do } \\
\text { Brasil }\end{array}$ & Peralta et al. 8862 \\
\hline $\begin{array}{l}\text { Lejeunea cristulata (Steph.) M.E. } \\
\text { Reiner \& Goda }\end{array}$ & MA & Moderada & $\begin{array}{c}\text { Endêmica do } \\
\text { Brasil }\end{array}$ & Peralta et al. 8873 \\
\hline Lejeunea deplanata Mitt. & $\begin{array}{c}\text { AM, CA, CE, MA, } \\
\text { PN }\end{array}$ & Ampla & Neotropical & $\begin{array}{c}\text { Peralta \& Carmo } \\
14212\end{array}$ \\
\hline Lejeunea filipes Spruce & MA, PN & Rara & Neotropical & $\begin{array}{c}\text { Peralta \& Carmo } \\
14203\end{array}$ \\
\hline Lejeunea flava (Sw.) Nees & $\begin{array}{l}\text { AM, CA, CE, MA, } \\
\text { PM, PN }\end{array}$ & Ampla & Pantropical & $\begin{array}{l}\text { Peralta \& Gugliota } \\
\text { s.n. (SP 437721) }\end{array}$ \\
\hline $\begin{array}{l}\text { Lejeunea grossitexta (Steph.) M.E. } \\
\text { Reiner \& Goda }\end{array}$ & MA & Moderada & Neotropical & $\begin{array}{c}\text { Peralta \& Carmo } \\
14192\end{array}$ \\
\hline Lejeunea herminieri (Steph.) R.L. Zhu & MA & Rara & Neotropical & $\begin{array}{c}\text { Peralta \& Carmo } \\
14692\end{array}$ \\
\hline $\begin{array}{l}\text { Lejeunea laeta (Lehm. \& Lindenb.) } \\
\text { Lehm. \& Lindenb. }\end{array}$ & MA & Moderada & Neotropical & $\begin{array}{c}\text { Peralta \& Gugliota } \\
13524\end{array}$ \\
\hline Lejeunea laetevirens Nees \& Mont. & $\begin{array}{l}\mathrm{AM}, \mathrm{CA}, \mathrm{CE}, \mathrm{MA}, \\
\text { PN }\end{array}$ & Ampla & América tropical & $\begin{array}{c}\text { Peralta \& Carmo } \\
14260\end{array}$ \\
\hline Lejeunea oligoclada Spruce & MA & Moderada & $\begin{array}{l}\text { Endêmica do } \\
\text { Brasil }\end{array}$ & $\begin{array}{c}\text { Peralta \& Gugliota } \\
13713\end{array}$ \\
\hline
\end{tabular}


Tabela 1 (continuação)

\begin{tabular}{|c|c|c|c|c|}
\hline Táxons (Divisão/Família/Espécie) & $\begin{array}{c}\text { Domínio } \\
\text { Fitogeográfico }\end{array}$ & $\begin{array}{l}\text { Distribuição } \\
\text { Brasil }\end{array}$ & $\begin{array}{l}\text { Distribuição } \\
\text { mundial }\end{array}$ & Voucher \\
\hline Lejeunea phyllobola Nees \& Mont. & $\begin{array}{l}\text { AM, CA, CE, MA, } \\
\text { PN }\end{array}$ & Ampla & América tropical & $\begin{array}{l}\text { Peralta \& Carmo } \\
14225\end{array}$ \\
\hline Lejeunea puiggariana & MA, PN & Moderada & Neotropical & $\begin{array}{c}\text { Peralta \& Gugliota } \\
13448\end{array}$ \\
\hline Lejeunea raddiana Lindenb. & MA & Rara & Neotropical & $\begin{array}{l}\text { Peralta \& Carmo } \\
\quad 14767\end{array}$ \\
\hline $\begin{array}{l}\text { Lepidolejeunea involuta (Gottsche) } \\
\text { Grolle }\end{array}$ & AM, MA & Moderada & América tropical & $\begin{array}{c}\text { Peralta \& Carmo } \\
14530\end{array}$ \\
\hline Leptolejeunea brasiliensis Bischl. & MA & Moderada & $\begin{array}{l}\text { América do Sul } \\
\text { tropical }\end{array}$ & $\begin{array}{c}\text { Peralta \& Gugliota } \\
13527\end{array}$ \\
\hline $\begin{array}{l}\text { Leptolejeunea elliptica (Lehm. \& } \\
\text { Lindenb.) Schiffn. }\end{array}$ & $\mathrm{AM}, \mathrm{CA}, \mathrm{CE}, \mathrm{MA}$ & Ampla & América tropical & Peralta et al. 8898 \\
\hline $\begin{array}{l}\text { Leptolejeunea exocellata (Spruce) A. } \\
\text { Evans }\end{array}$ & $\mathrm{AM}, \mathrm{CE}, \mathrm{MA}, \mathrm{PN}$ & Ampla & América tropical & $\begin{array}{c}\text { Peralta \& Carmo } \\
14154\end{array}$ \\
\hline $\begin{array}{l}\text { Lopholejeunea nigricans (Lindenb.) } \\
\text { Schiffn. }\end{array}$ & $\mathrm{AM}, \mathrm{CE}, \mathrm{MA}, \mathrm{PN}$ & Ampla & Pantropical & Peralta et al. 9325 \\
\hline Lopholejeunea subfusca (Nees) Schiffn. & $\begin{array}{l}\mathrm{AM}, \mathrm{CA}, \mathrm{CE}, \mathrm{MA} \\
\text { PN }\end{array}$ & Ampla & Neotropical & $\begin{array}{c}\text { Peralta \& Carmo } \\
14538\end{array}$ \\
\hline Marchesinia brachiata (Sw.) Schiffn. & $\mathrm{AM}, \mathrm{CE}, \mathrm{MA}$ & Ampla & $\begin{array}{l}\text { América tropical e } \\
\text { África }\end{array}$ & Peralta et al. 9323 \\
\hline *Microlejeunea acutifolia Steph. & $\mathrm{AM}$ & Rara & América tropical & $\begin{array}{c}\text { Peralta \& Gugliota } \\
13759\end{array}$ \\
\hline Microlejeunea bullata (Taylor) Steph. & $\begin{array}{l}\mathrm{AM}, \mathrm{CA}, \mathrm{CE}, \mathrm{MA} \\
\text { PM, PN }\end{array}$ & Ampla & América tropical & $\begin{array}{c}\text { Peralta \& Carmo } \\
14575\end{array}$ \\
\hline Microlejeunea epiphylla Bischl. & $\begin{array}{c}\mathrm{AM}, \mathrm{CA}, \mathrm{CE}, \mathrm{MA} \\
\text { PN }\end{array}$ & Ampla & Neotropical & $\begin{array}{c}\text { Peralta \& Carmo } \\
14449\end{array}$ \\
\hline $\begin{array}{l}\text { Neurolejeunea breutelii (Gottsche) A. } \\
\text { Evans }\end{array}$ & MA & Moderada & $\begin{array}{c}\text { América tropical e } \\
\text { EUA }\end{array}$ & Peralta et al. 8984 \\
\hline $\begin{array}{l}\text { Odontolejeunea decemdentata (Spruce) } \\
\text { Steph. }\end{array}$ & MA & Rara & América tropical & $\begin{array}{c}\text { Peralta \& Gugliota } \\
13447\end{array}$ \\
\hline $\begin{array}{l}\text { Odontolejeunea lunulata (F. Weber) } \\
\text { Schiffn. }\end{array}$ & $\mathrm{AM}, \mathrm{CE}, \mathrm{MA}$ & Ampla & $\begin{array}{l}\text { América tropical e } \\
\text { África }\end{array}$ & Peralta et al. 9330 \\
\hline Prionolejeunea aemula Steph. & AM, CE, MA & Moderada & América tropical & $\begin{array}{c}\text { Peralta \& Carmo } \\
14504\end{array}$ \\
\hline Pycnolejeunea contigua (Nees) Grolle & AM, MA & Ampla & Pantropical & $\begin{array}{c}\text { Peralta \& Gugliota } \\
13771\end{array}$ \\
\hline $\begin{array}{l}\text { Pycnolejeunea densistipula (Lehm. \& } \\
\text { Lindenb.) Steph. }\end{array}$ & MA & Rara & América tropical & $\begin{array}{c}\text { Peralta \& Gugliota } \\
13871\end{array}$ \\
\hline $\begin{array}{l}\text { Rectolejeunea berteroana (Gottsche ex } \\
\text { Steph.) A. Evans }\end{array}$ & AM, MA & Ampla & América tropical & $\begin{array}{c}\text { Peralta \& Carmo } \\
14328\end{array}$ \\
\hline $\begin{array}{l}\text { Rectolejeunea emarginuliflora } \\
\text { (Gottsche ex Schiffn.) A. Evans }\end{array}$ & MA & Rara & América tropical & $\begin{array}{c}\text { Peralta \& Gugliota } \\
13629\end{array}$ \\
\hline $\begin{array}{l}\text { Schiffneriolejeunea polycarpa (Nees) } \\
\text { Gradst. }\end{array}$ & $\begin{array}{l}\mathrm{AM}, \mathrm{CA}, \mathrm{CE}, \mathrm{MA} \\
\text { PN }\end{array}$ & Ampla & Pantropical & Spielmann et al. 4835 \\
\hline Taxilejeunea isocalycina (Nees) Steph. & AM, MA & Moderada & América tropical & $\begin{array}{c}\text { Peralta \& Carmo } \\
14741\end{array}$ \\
\hline
\end{tabular}


Tabela 1 (continuação)

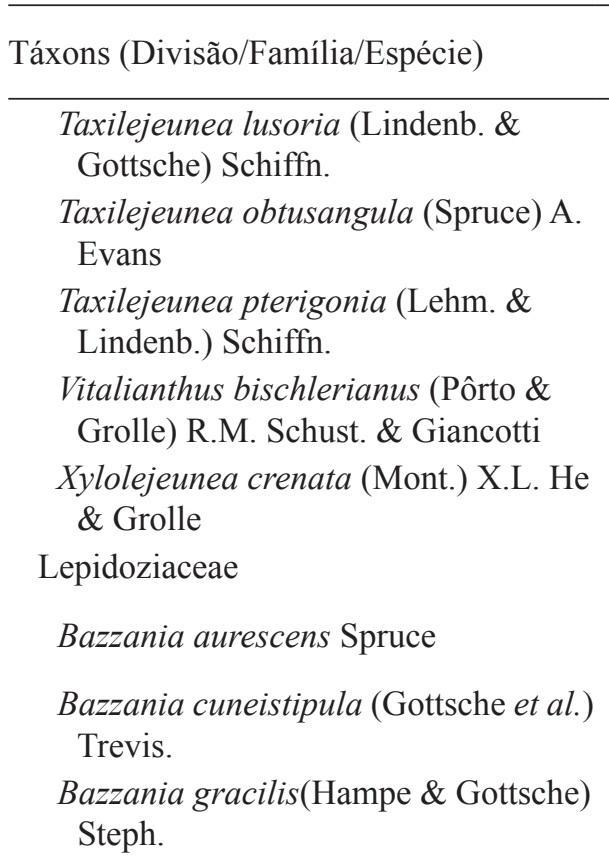

Bazzania heterostipa (Steph.) Fulford

Bazzania hookeri (Lindenb.) Trevis.

*Bazzania pallidevirens (Steph.) Fulford.

Bazzania phyllobola Spruce

Kurzia capillaris (Sw.) Grolle

Paracromastigum pachyrhizum (Nees) Fulford

Telaranea diacantha (Mont.) J.J. Engel \& G.L. Merr.

Telaranea nematodes (Gottshe ex Austin) M. Howe. Bull.

Lophocoleaceae

Chiloscyphus lindmannii (Steph.) J.J. Engel \& R.M. Schust.

*Chiloscyphus mandonii (Steph.) J.J.

Engel \& R.M. Schust.

Chiloscyphus martianus (Nees) J.J.

Engel \& R.M. Schust.

Chiloscyphus muricatus (Lehm.) J.J.

Engel \& R.M. Schust.

Heteroscyphus amphibolius (Nees)

Schiffn.

Leptoscyphus porphyrius (Nees) Grolle

Lophocolea bidentata (L.) Dumort.

Metzgeriaceae

Metzgeria albinea Spruce
Domínio Distribuição Distribuição

Fitogeográfico Brasil mundial

\begin{tabular}{|c|c|c|c|}
\hline AM, MA & Moderada & América tropical & $\begin{array}{c}\text { Peralta \& Carmo } \\
14641\end{array}$ \\
\hline AM, MA & Moderada & América tropical & $\begin{array}{c}\text { Peralta \& Carmo } \\
14810\end{array}$ \\
\hline MA & Moderada & América tropical & $\begin{array}{c}\text { Peralta \& Gugliota } \\
13755\end{array}$ \\
\hline MA & Moderada & $\begin{array}{l}\text { Endêmica do } \\
\text { Brasil }\end{array}$ & $\begin{array}{c}\text { Peralta \& Carmo } \\
14236\end{array}$ \\
\hline AM, MA & Ampla & América tropical & $\begin{array}{c}\text { Peralta \& Gugliota } \\
13599\end{array}$ \\
\hline
\end{tabular}

AM, CE, MA Moderada América tropical

Peralta \& Gugliota 13539

MA

Rara

América tropical

Peralta \& Gugliota 13642

AM, MA Moderada América tropical

Endêmica do Brasil

MA Moderada

AM, MA

Ampla

América tropical

América do Sul tropical

AM, MA Moderada Neotropical

AM, CE, MA Ampla

América tropical e África

Moderada

América tropical

Carmo \& Peralta 706

AM, CE, MA Ampla Pantropical

Peralta \& Carmo 14773

AM, CE, MA Ampla Pantropical

Carmo \& Peralta 670

AM, CE, MA Moderada

América do Sul tropical

Peralta et al. 8940

MA

Rara

AM, CE, MA, PN

Ampla

MA

Moderada

América do Sul tropical

América tropical e África

Cosmopolita

Carmo \& Peralta 669

Carmo \& Peralta 766

Peralta \& Carmo 14202

MA, CE Moderada América tropical

Peralta et al. 9400

AM, MA Moderada América tropical

Peralta et al. 9349

AM, CE, MA Moderada Subcosmopolita

Peralta et al. 9011

CE, MA Ampla Pantropical Carmo \& Peralta 566 
Tabela 1 (continuação)

\begin{tabular}{|c|c|c|c|c|}
\hline Táxons (Divisão/Família/Espécie) & $\begin{array}{c}\text { Domínio } \\
\text { Fitogeográfico } \\
\end{array}$ & $\begin{array}{c}\text { Distribuição } \\
\text { Brasil }\end{array}$ & $\begin{array}{c}\text { Distribuição } \\
\text { mundial }\end{array}$ & Voucher \\
\hline Metzgeria conjugata Lindb. & MA & Moderada & Subcosmopolita & $\begin{array}{c}\text { Peralta \& Carmo } \\
14807\end{array}$ \\
\hline $\begin{array}{l}\text { Metzgeria decipiens (C. Massal.) } \\
\text { Schiffn. }\end{array}$ & MA & Moderada & Pantropical & $\begin{array}{c}\text { Peralta \& Carmo } \\
14753\end{array}$ \\
\hline Metzgeria dichotoma (Sw.) Nees & MA, CE & Moderada & América tropical & Peralta et al. 9408 \\
\hline Metzgeria furcata (L.) Dumort. & $\mathrm{AM}, \mathrm{CE}, \mathrm{MA}$ & Ampla & Subcosmopolita & $\begin{array}{c}\text { Peralta \& Gugliota } \\
13689\end{array}$ \\
\hline Metzgeria myriopoda Lindb. & CE, MA & Moderada & América tropical & $\begin{array}{c}\text { Peralta \& Carmo } \\
14187\end{array}$ \\
\hline Metzgeria schyphigera A. Evans & MA & Rara & América tropical & Carmo \& Peralta 651 \\
\hline Metzgeria uncigera A. Evans & MA & Moderada & América tropical & Carmo \& Peralta 624 \\
\hline \multicolumn{5}{|l|}{ Pallaviciniaceae } \\
\hline Pallavicinia lyellii (Hook.) Gray & AM, CE, MA, PN & Ampla & Cosmopolita & Peralta et al. 8969 \\
\hline na aspera Steph. & AM, CE, MA, PN & Ampla & América tropical & $\begin{array}{c}\text { Peralta \& Gugliota } \\
13426\end{array}$ \\
\hline $\begin{array}{l}\text { Symphyogyna brasiliensis (Nees) Nees } \\
\text { \& Mont. }\end{array}$ & $\mathrm{AM}, \mathrm{CE}, \mathrm{MA}$ & Ampla & Neotropical & $\begin{array}{c}\text { Peralta \& Carmo } \\
14361\end{array}$ \\
\hline $\begin{array}{l}\text { Symphyogyna podophylla (Thunb.) } \\
\text { Mont. \& Nees }\end{array}$ & MA & Moderada & $\begin{array}{c}\text { América tropical e } \\
\text { África }\end{array}$ & Peralta et al. 9396 \\
\hline \multicolumn{5}{|l|}{ Plagiochilaceae } \\
\hline $\begin{array}{l}\text { Plagiochila adianthoides (Sw.) } \\
\text { Lindenb. }\end{array}$ & CE, MA & Moderada & América tropical & Peralta et al. 9362 \\
\hline Plagiochila bifaria (Sw.) Lindenb. & AM, MA & Moderada & América tropical & Carmo \& Peralta 642 \\
\hline Plagiochila bryopterioides Spruce & MA & Rara & América tropical & $\begin{array}{c}\text { Peralta \& Gugliota } \\
13853\end{array}$ \\
\hline $\begin{array}{l}\text { Plagiochila corrugata (Nees) Nees \& } \\
\text { Mont. }\end{array}$ & $\mathrm{AM}, \mathrm{CE}, \mathrm{MA}$ & Ampla & América tropical & Peralta et al. 8879 \\
\hline Plagiochila cristata (Sw.) Lindenb. & AM, MA & Moderada & América tropical & Peralta et al. 9278 \\
\hline Plagiochila exigua (Taylor) Taylor & MA & Rara & $\begin{array}{l}\text { América tropical e } \\
\text { África }\end{array}$ & $\begin{array}{c}\text { Peralta \& Carmo } \\
14688\end{array}$ \\
\hline $\begin{array}{l}\text { Plagiochila gymnocalycina (Lehm. \& } \\
\text { Lindenb.) Mont. }\end{array}$ & MA & Moderada & América tropical & $\begin{array}{c}\text { Peralta \& Carmo } \\
14496\end{array}$ \\
\hline Plagiochila macrostachya Lindenb. & AM, MA & Moderada & América tropical & $\begin{array}{c}\text { Peralta \& Gugliota } \\
13812\end{array}$ \\
\hline Plagiochila montagnei Nees & AM, MA & Ampla & América tropical & $\begin{array}{c}\text { Peralta \& Gugliota } \\
13698\end{array}$ \\
\hline Plagiochila patentissima Lindenb. & MA & Ampla & América tropical & $\begin{array}{c}\text { Peralta \& Gugliota } \\
13660\end{array}$ \\
\hline Plagiochila patula (Sw.) Lindenb. & AM, MA & Moderada & América tropical & $\begin{array}{c}\text { Peralta \& Carmo } \\
14501\end{array}$ \\
\hline Plagiochila raddiana Lindenb. & $\mathrm{AM}, \mathrm{CE}, \mathrm{MA}$ & Ampla & América tropical & $\begin{array}{c}\text { Peralta \& Gugliota } \\
13419\end{array}$ \\
\hline Plagiochila rutilans Lindenb. & $\mathrm{AM}, \mathrm{CE}, \mathrm{MA}$ & Ampla & América tropical & $\begin{array}{c}\text { Peralta \& Carmo } \\
14152\end{array}$ \\
\hline Plagiochila simplex (Sw.) Lindenb. & $\mathrm{AM}, \mathrm{CE}, \mathrm{MA}$ & Ampla & América tropical & $\begin{array}{c}\text { Peralta \& Carmo } \\
14818\end{array}$ \\
\hline
\end{tabular}


Tabela 1 (continuação)

\begin{tabular}{|c|c|c|c|c|}
\hline Táxons (Divisão/Família/Espécie) & $\begin{array}{c}\text { Domínio } \\
\text { Fitogeográfico } \\
\end{array}$ & $\begin{array}{c}\text { Distribuição } \\
\text { Brasil }\end{array}$ & $\begin{array}{c}\text { Distribuição } \\
\text { mundial }\end{array}$ & Voucher \\
\hline $\begin{array}{l}\text { Plagiochila subplana Lindenb. } \\
\text { Porellaceae }\end{array}$ & AM, MA & Ampla & América tropical & Carmo \& Peralta 638 \\
\hline Porella brasiliensis (Raddi) Schiffn. & $\mathrm{MA}, \mathrm{CE}$ & Moderada & $\begin{array}{l}\text { América do Sul } \\
\text { tropical }\end{array}$ & Peralta et al. 9322 \\
\hline $\begin{array}{l}\text { Porella swartziana (Weber) Trevis. } \\
\text { Radulaceae }\end{array}$ & $\mathrm{MA}, \mathrm{CE}$ & Moderada & América tropical & Peralta et al. 9302 \\
\hline Radula angulata Steph. & $\mathrm{CE}, \mathrm{MA}$ & Moderada & América tropical & $\begin{array}{c}\text { Peralta \& Gugliota } \\
13887\end{array}$ \\
\hline Radula brasilica Yamada & MA & Rara & $\begin{array}{l}\text { Endêmica do } \\
\text { Brasil }\end{array}$ & $\begin{array}{c}\text { Peralta \& Gugliota } \\
13555\end{array}$ \\
\hline Radula decora Gottsche ex Steph. & MA & Rara & $\begin{array}{l}\text { América do Sul } \\
\text { tropical }\end{array}$ & Peralta et al. 9410 \\
\hline Radula fendleri Gottsche ex Steph. & MA & Rara & $\begin{array}{l}\text { América do Sul } \\
\text { tropical }\end{array}$ & $\begin{array}{c}\text { Peralta \& Carmo } \\
14815\end{array}$ \\
\hline *Radula gottscheana Taylor & MA & Rara & América tropical & $\begin{array}{c}\text { Peralta \& Gugliota } \\
13515\end{array}$ \\
\hline Radula javanica Gottsche & $\mathrm{AM}, \mathrm{CE}, \mathrm{MA}, \mathrm{PN}$ & Ampla & Pantropical & Carmo \& Peralta 588 \\
\hline Radula mammosa Spruce & AM, MA & Rara & América tropical & Carmo \& Peralta 644 \\
\hline Radula mexicana Lindenb. & AM, MA & Moderada & $\begin{array}{l}\text { América tropical, } \\
\text { África e Europa }\end{array}$ & Peralta et al. 8989 \\
\hline Radula nudicaulis Steph. & MA & Moderada & $\begin{array}{l}\text { América do } \\
\text { sul tropical e } \\
\text { Macronésia }\end{array}$ & $\begin{array}{c}\text { Peralta \& Carmo } \\
14397\end{array}$ \\
\hline Radula quadrata Gottsche & AM, MA & Moderada & Cosmopolita & $\begin{array}{c}\text { Peralta \& Carmo } \\
14221\end{array}$ \\
\hline Radula recubans Taylor & AM, MA & Ampla & Américas & Peralta et al. 8931 \\
\hline Radula schaefer-verwimpii Yamada & MA & Rara & $\begin{array}{l}\text { América do Sul } \\
\text { tropical }\end{array}$ & Carmo \& Peralta 523 \\
\hline Radula sinuata Gottsche ex Steph. & MA & Moderada & $\begin{array}{l}\text { América do Sul } \\
\text { tropical }\end{array}$ & Peralta et al. 9033 \\
\hline Radula tenera Mitt. ex Steph. & MA & Rara & $\begin{array}{l}\text { América do Sul } \\
\text { tropical }\end{array}$ & $\begin{array}{c}\text { Peralta \& Carmo } \\
14453\end{array}$ \\
\hline Radula voluta Taylor ex Gottsche & MA & Moderada & $\begin{array}{l}\text { América tropical, } \\
\text { África e Europa }\end{array}$ & $\begin{array}{c}\text { Peralta \& Carmo } \\
14243\end{array}$ \\
\hline
\end{tabular}

destas, $152(40 \%)$ foram registradas exclusivamente para o domínio da Mata Atlântica do Brasil. As espécies que ainda não tinham sido classificadas como ocorrentes dentro desse domínio foram Cheilolejeunea beyrichii (Lindenb.) Reiner, a qual era encontrada apenas na Caatinga e no Cerrado brasileiro, e é endêmica do Brasil; as espécies Lepidopilum pallidonitens (Müll. Hal.) Paris e Microlejeunea acutifolia Steph., que até então tinham sido encontradas apenas na Amazônia e que são novas ocorrências para o Estado de São Paulo e Bryum leptocladon Sull., a qual só era conhecida para o Cerrado e também se caracteriza como uma nova ocorrência, todas essas espécies citadas anteriormente apresentam uma distribuição rara, ou restrita, para o Brasil (tabela 1) (Costa et al. 2011, Costa \& Peralta 2015).

Os dados apresentados nesse trabalho de levantamento florístico para o Núcleo Santa Virgínia acrescentaram informações relevantes sobre a diversidade, ocorrência e distribuição das briófitas na Mata Atlântica para o estado de São Paulo. O Parque Estadual da Serra do Mar, Núcleo Santa Virgínia, apresentou 1/4 da diversidade de briófitas estimadas para o país inteiro e um número significativo de espécies endêmicas e novas ocorrências para o 
Tabela 2. Relação da distribuição e da riqueza de espécies encontradas no Núcleo de Santa Virgínia com outras escalas regionais. Os números entre parênteses representam a porcentagem relacionada com a riqueza encontrada no Parque Estadual da Serra do Mar, Núcleo de Santa Virgínia, Estado de São Paulo, Brasil.

Table 2. Distribution and richness of species found in the Núcleo Santa Virgínia in relation to other regional scales. The numbers in parentheses represent the percentage related to the richness found in the Parque Estadual da Serra do Mar, Núcleo Santa Virgínia, São Paulo State, Brazil

\begin{tabular}{lcc}
\hline Região & Riqueza de briófitas & Referências \\
\hline Núcleo de Santa Virgínia & 386 & Carmo et al., dados não publicados \\
Estado de São Paulo & $900(43 \%)$ & Costa \& Peralta 2015 \\
Brasil & $1524(25 \%)$ & Costa \& Peralta 2015 \\
América Tropical & $3980(10 \%)$ & Gradstein et al. 2001 \\
\hline
\end{tabular}

\section{Riqueza}

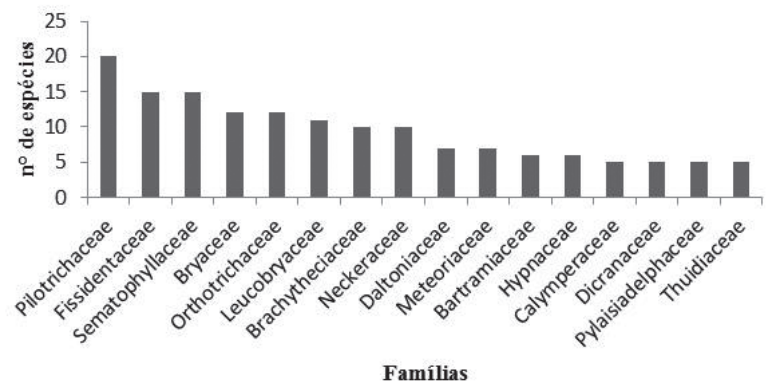

Figura 1. Riqueza das espécies de musgos (Bryophyta) por famílias que apresentaram cinco ou mais espécies.

Figure 1. Richness of families mosses species (Bryophyta) which have five or more species.

Estado, o que revela a importância dessa unidade de conservação para a proteção da biodiversidade de briófitas na Mata Atlântica, por outro lado, esse trabalho revela a importância dos trabalhos de inventários florísticos e a necessidade de estudos de briófitas com enfoque para outras áreas como a ecologia, fitogeografia e diversidade genética.

\section{Agradecimentos}

Ao Instituto de Botânica de São Paulo (Ibt) por fornecer a assistência e estrutura necessária para a realização desse trabalho. À organização e administração do Parque Estadual da Serra do Mar, Núcleo Santa Virgínia, pela hospedagem e auxílio de campo e aos colegas Dra. Adriana Gugliota, Msc. Ricardo Matheus Pires e Cecília Mayumi pela companhia e auxílio durante as coletas.

\section{Literatura citada}

Ballejos, J. \& Bastos, C.J.P. 2009. Musgos Pleurocárpicos do Parque Estadual das Sete Passagens, Miguel Calmon, Bahia, Brasil. Hoehnea 36: 479-495.

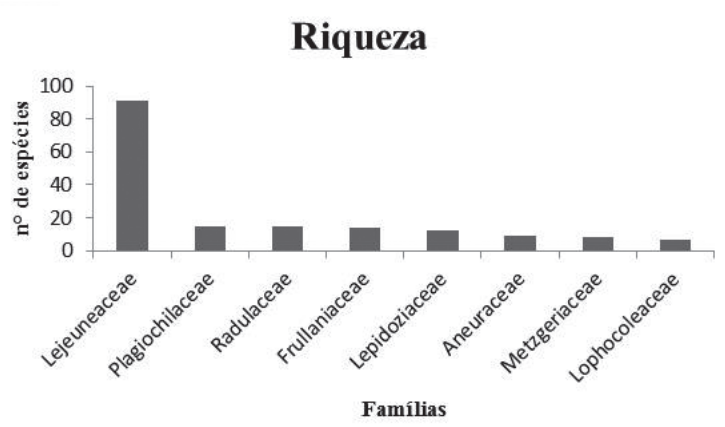

Figura 2. Riqueza das espécies de hepáticas (Marchantiophyta) por famílias que apresentaram cinco ou mais espécies.

Figure 2. Richness of families liverworts species (Marchantiophyta) which have five or more species.

Bastos, C.J.P. \& Vilas Bôas-Bastos, S.B. 2008. Musgos Acrocárpicos e Cladocárpicos (Bryophyta) da Reserva Ecológica da Michelin, Igrapiúna, Bahia, Brasil. Stientibus Série Ciências Biológicas 8: 275-279.

Bastos, C.J.P., Yano, O. \& Vilas Bôas-Bastos, S.B. 2000. Briófitas de Campos rupestres da Chapada Diamantina, Estado da Bahia, Brasil. Revista Brasileira de Botânica 23: 357-368.

Bordin, J. \& Yano, O. 2013. Fissidentaceae (Bryophyta) do Brasil. Boletim do Instituto de Botânica 22: 1-72.

Buck, W.R. 1998. Pleurocarpous Mosses of the West Indies. Memoirs of The New York Botanical Garden 1: 1-401.

Câmara, P.E.A.S. 2008a. Musgos pleurocárpicos das matas de galeria da Reserva Ecológica do IBGE, RECOR, Distrito Federal, Brasil. Acta Botanica Brasilica 22: 573-581.

Câmara, P.E.A.S. 2008b. Musgos acrocárpicos das Matas de Galeria da Reserva Ecológica do IBGE, RECOR, Distrito Federal, Brasil. Acta Botanica Brasilica 22: 1027-1035.

Câmara, P.E.A.S. \& Costa, D.P. 2006. Hepáticas e antóceros das matas de galeria da Reserva Ecológica do IBGE, RECOR, Distrito Federal, Brasil. Hoehnea 33: 79-87. 
Costa, D.P. \& Lima, F.M. 2005. Moss diversity in the tropical rainforests of Rio de Janeiro, Southeastern Brazil. Revista Brasileira de Botânica 28: 671-685.

Costa, D.P. \& Santos, N.D. 2009. Conservação de hepáticas na Mata Atlântica do sudeste do Brasil: uma análise regional no Estado do Rio de Janeiro. Acta Botanica Brasilica 23: 913-922.

Costa, D.P. \& Peralta, D.F. 2015. Briófitas In: Lista de Espécies da Flora do Brasil. Jardim Botânico do Rio de Janeiro. Disponível em http://floradobrasil. jbrj.gov.br/jabot/floradobrasil/FB128472 (acesso em 12-XI-2015).

Costa, D.P., Bastos, C.J.P. \& Schäfer-Verwimp, A. 2015. Lejeuneaceae In: Lista de Espécies da Flora do Brasil. Jardim Botânico do Rio de Janeiro. Disponível em http://www.floradobrasil.jbrj.gov.br/jabot/floradobrasil/ FB97355. (acesso em 12-XI-2015).

Costa, D.P., Pôrto, K.C., Luizi-Ponzo, A.P., Ilkiu-Borges, A.L., Bastos, C.J.P., Câmara, P.E.A.S., Peralta, D.F., Bôas-Bastos, S.B.V., Imbassahy, C.A.A., Henriques, D.K., Gomes, H.C.S., Rocha, L.M., Santos, N.D., Siviero, T. S., Vaz-Imbassahy, T.F. \& Churchill, S.P. 2011. Synopsis of the Brazilian moss flora: checklist, distribution and conservation. Nova Hedwigia 93: 277-334.

Costa, D.P., Santos, N.D., Rezende, M.A., Buck, W.R. \& Schäfer-Verwimp, A. 2015. Bryoflora of the Itatiaia National Park along an elevation gradient: diversity and conservation. Biodiversity and Conservation.

Crandall-Stotler, B., Stotler, R.E. \& Long, D.G. 2009. Morphology and classification of the Marchantiophyta. In: B. Goffinet \& A.J. Shaw Bryophyte Biology. 2 ed. Cambdrige University Press, pp. 1-54.

Forzza, R.C., Leitman, P.M., Costa, A.F., Carvalho, J.R., Peixoto, A.L., Walter, B.M.T., Bicudo, C., Zappi, D., Costa, D.P., Lleras, E., Martinelli, G., Lima, H.C., Prado, J., Stehmann, J.R., Baumgratz, J.F.A., Pirani, J.R., Sylvestre, L., Maia, L.C., Lohmann, L.G., Queiroz, L.P., Silveira, M., Coelho, M.N., Mamede, M.C., Bastos, M.N.C., Morin, M.P., Barbosa, M.R., Menezes, M., Hopkins, M., Secco, R., Cavalcanti, T.B. \& Souza, V.C. 2010. Introdução. In: Lista de espécies da Flora do Brasil. Jardim Botânico do Rio de Janeiro. v. 1. Jardim Botânico do Rio de Janeiro, pp. 452 - 521.

Frahm, J.P. 1991. Dicranaceae: Campylopodioideae, Paraleucobryoideae. Flora Neotropica Monograph 54: 1-237.

Fundação SOS Mata Atlântica. 2015. Mata Atlântica. Disponível em https://www.sosma.org.br/nossa-causa/ a-mata-atlantica/ (acesso em 12-XI-2015).

Goffinet, B., Buck, W.R. \& Shaw, A.J. 2009. Morphology, anatomy and classification of the Bryophyta. In: B. Goffinet \& A.J. Shaw. Bryophyte Biology. 2 ed. Cambdrige University Press, pp. 56-138.
Gradstein, S.R. \& Costa, D.P. 2003. The Hepaticae and Anthocerotae of Brazil. Memoirs of The New York Botanical Garden 87: 1-318.

Gradstein, S.R., Churchill, S.P. \& Salazar-Allen, N. 2001. Guide to the Bryophytes of Tropical America. Memoirs of The New York Botanical Garden 86: 1-577.

Instituto Ekos Brasil. 2006. Plano de Manejo Parque Estadual da Serra do Mar. Disponível em http://www. ekosbrasil.org/anexos/1.\%20Resumo\%20Executivo.pdf (acesso em 12-XI-2015).

Peralta, D.F. \& Yano, O. 2008. Briófitas do Parque Estadual da Ilha Anchieta, Ubatuba, estado de São Paulo, Brasil. Iheringia 63: 101-127.

Peralta, D.F. \& Yano, O. 2012. Briófitas da Serra do Itapeti. In: M.S.C. Morini, \& V.F.O. Miranda (org.). Serra do Itapeti: Aspectos Históricos, Sociais e Naturalísticos. Santa Cruz do Rio Pardo: Viena Grafica e Editora, v. 1. pp. 1-397.

Pires, R.M. 2015. Políporos (Basidiomycota) do núcleo de Santa Virgínia do Parque Estadual da Serra do Mar, SP, Brasil. Dissertação de Mestrado, Instituto de Botânica, São Paulo.

Reis, L.C., Oliveira, H.C. \& Bastos, C.J.P. 2015. Hepáticas (Marchantiophyta) epífitas de duas áreas de Floresta Atlântica no estado da Bahia, Brasil. Pesquisas, série botânica 67: 225-241.

Renzaglia, K.S., Villarreal, J.C. \& Duff, R.J. 2009. New insights into morphology, anatomy and systematics of hornworts. In: B. Goffinet \& A.J. Shaw. Bryophyte Biology. Second Edition. Cambdrige University Press, pp. 139-171.

Santos, N.D. \& Costa, D.P. 2008. A importância de Reservas Particulares do Patrimônio Natural para a conservação da brioflora da Mata Atlântica: um estudo em El Nagual, Magé, RJ, Brasil. Acta Botanica Brasilica 22: 359-372.

Santos, N.D. \& Costa, D.P. 2010a. Phytogeography of the liverwort flora of the Atlantic Forest of southeastern Bazil. Journal of Bryology 32: 9-22.

Santos, N.D. \& Costa, D.P. 2010b. Altitudinal zonation of liverworts in the Atlantic Forest, Southeastern Brazil. The Bryologist 113: 631-645.

Secretaria de Estado de Meio Ambiente. 2015. O Parque Estadual da Serra do Mar. Núcleo de Santa Virgínia. Disponível em http://www.ambiente.sp.gov.br/parqueserra-do-mar-nucleo-santa-virginia/sobre-o-parque/ (acesso em 12-XI-2015).

Silva, M.P.P. \& Pôrto, K.C. 2010. Spatial structure of bryophyte communities along an edge-interior gradient in an Atlantic Forest remnant in Northeast Brazil. Journal of Bryology 32: 101-112.

Silva, M.P.P. \& Pôrto, K.C. 2015. Diversity of bryophytes in priority areas for conservation in the Atlantic Forest of northeast Brazil. Acta Botanica Brasilica 29: 16-23. 
Sharp, A.J., Crum, H. \& Eckel, P. 1994. The Moss Flora of Mexico. Memoirs of The New York Botanical Garden 69: 1-1113.

Valente, E.B. \& Pôrto, K.C. 2006. Hepáticas (Marchantiophyta) de um fragmento de Mata Atlântica na Serra da Jibóia, município de Santa Terezinha, BA, Brasil. Acta Botanica Brasilica 20: 433-441.

Valente, E.B., Pôrto, K.C., Vilas Bôas-Bastos, S.B. \& Bastos, C.J.P. 2009. Musgos (Bryophyta) de um fragmento de Mata Atlântica na Serra da Jibóia, município de Santa Terezinha, BA, Brasil. Acta Botanica Brasilica 23: 369-375.

Vaz, T.F. \& Costa, D.P. 2006. Os gêneros Lepidopilidium, Lepidopilum, Pilotrichum e Thamniopsis (Pilotrichaceae, Bryophyta) no Estado do Rio de Janeiro, Brasil. Acta Botanica Brasilica 20: 975-993.

Visnadi, S.R. 2002. Meteoriaceae (Bryophyta) da Mata Atlântica do estado de São Paulo. Hoehnea 29: 159-187.

Visnadi, S.R. 2004. Briófitas de praias do Estado de São Paulo, Brasil. Acta Botanica Brasilica 18: 91-97.

Visnadi, S.R. 2005. Brioflora da Mata Attântica do estado de São Paulo: região norte. Hoehnea 32: 215-231.

Visnadi, S.R. 2006. Sematophyllaceae da Mata Attântica do nordeste do Estado de São Paulo. Hoehnea 33: 455-484.
Visnadi, S.R. 2009. Briófitas do Caxetal, em Ubatuba, São Paulo, Brasil. Tropical Bryology 30: 8-14.

Visnadi, S.R. 2013. Briófitas de áreas antrópicas do Parque Estadual da Serra do Mar, Núcleo Picinguaba, Ubatuba, estado de São Paulo, Brasil. Boletim do Museu Paraense Emilio Goeldi 8: 49-62.

Yano, O. 2008. Catálogo de antóceros e hepáticas brasileiros: literatura original, basiônimo, localidadetipo e distribuição geográfica. Boletim do Instituto de Botânica 19: 1-110.

Yano, O. \& Peralta, D.F. 2007. Briófitas da Ilha do Bom Abrigo, Estado de São Paulo, Brasil. Hoehnea 34: 87-94.

Yano, O. \& Peralta, D.F. 2008. Briófitas da Ilhabela, Estado de São Paulo, Brasil. Hoehnea 35: 111-121.

Yano, O. \& Peralta, D.F. 2009. Flora de Grão-Mogol, Minas Gerais. Briófitas (Bryophyta e Marchantiophyta). Boletim de Botânica da Universidade de São Paulo 27: 1-26.

Yano, O. \& Peralta, D.F. 2011. Flora da Serra do Cipó, Minas Gerais: Briófitas (Anthocerotophyta, Bryophyta e Marchantiophyta). Boletim de Botânica da Universidade de São Paulo, 29: 135-211. 